\title{
Intra-axonal translation of Khsrp mRNA slows axon regeneration by destabilizing localized mRNAs
}

Priyanka Patel ${ }^{1}$, Courtney Buchanan ${ }^{1}$, Amar N. Kar ${ }^{1}$, Seung Joon Lee ${ }^{1 *}$, Pabitra K. Sahoo ${ }^{1}$, Anatoly

Urisman ${ }^{2}$, Juan Oses-Prieto ${ }^{2}$, Michela Dell'Orco ${ }^{3}$, Devon E. Cassidy ${ }^{1 * *}$, Sharmina Miller ${ }^{1}$, Elizabeth Thames ${ }^{1}$, Terika P. Smith ${ }^{1}$, Matthew D. Zdradzinski ${ }^{1}$, Alma L. Burlingame ${ }^{2}$, Nora Perrone-Bizzozero ${ }^{3}$, and Jeffery L. Twiss ${ }^{1}$

${ }^{1}$ Department of Biological Sciences, University of South Carolina, Columbia, South Carolina 29208, USA

${ }^{2}$ Department of Pharmaceutical Sciences, University of California, San Francisco, California 94143, USA

${ }^{3}$ Department of Neurosciences, University of New Mexico School of Health Sciences, Albuquerque, New Mexico 87131, USA

* Current address: Biogen, Cambridge, Massachusetts USA

** Current address: University of Michigan School of Medicine, Ann Arbor, Michigan USA

Running Title: Axon regeneration slowed by axon-intrinsic RNA destabilization

Word Count: Abstract - 226, Introduction - 518, Results - 2600, Discussion - 1500 (est), Materials \& Methods - 3072.

Figures: 6 main +4 supplemental

Acknowledgements: This work was supported by grant awards from Wings for Life Spinal Cord Injury Research Foundation (WFL-US-09/18 to PP), National Institutes of Health (R01-NS089633 to JLT and NPB; K01-NS105879 to TPS), the Dr. Miriam and Sheldon G. Adelson Medical Research Foundation (to JLT and ALB), South Carolina Spinal Cord Injury Research Fund (2019 PD-02 to PKS), and SC EPSCoR Stimulus Grant Program (18-SR04 to JLT). JLT is the incipient South Carolina SmartState Chair in Childhood Neurotherapeutics at the University of South Carolina.

Conflict of Interest: The authors declare no financial conflicts. 


\section{ABSTRACT}

Proteins generated by localized mRNA translation in axons support nerve regeneration through retrograde injury signaling and localized axon growth mechanisms. RNA binding proteins (RBP) are needed for this and other aspects of post-transcriptional control of localized mRNAs, but only a limited number of axonal RBPs have been reported. We used a targeted mass spectrometry approach to profile the axonal RBPs in naïve, injured and regenerating PNS axons. We detected 76 axonal proteins that are reported to have RNA binding activity, with the levels of several of these axonal RBPs changing with axonal injury and regeneration. These axonal RBPs with altered axoplasm levels include KHSRP that we previously reported decreases neurite outgrowth in developing CNS neurons. We show that KHSRP levels rapidly increase in sciatic nerve axons after crush injury and remain elevated increasing in levels out to 28 days post-sciatic nerve crush injury. Khsrp mRNA localizes into axons and the rapid increase in axonal KHSRP after axotomy is mediated by the local translation of its mRNA. KHSRP binds to mRNAs with a 3'UTR AU-rich element and targets those mRNAs to the cytoplasmic exosome for degradation. KHSRP knockout mice show increased axonal levels of defined KHSRP target mRNAs, Gap43 and Snap25 mRNAs, following sciatic nerve injury and accelerated nerve regeneration in vivo. These data indicate that axonal translation of Khsrp mRNA following nerve injury serves to destabilize other axonal mRNAs and slow axon regeneration.

Keywords: Axonal translation, mRNA stability, RNA binding protein, axon regeneration, conditioning nerve lesion 


\section{INTRODUCTION}

Localization of mRNAs to subcellular regions provides polarized cells with means to rapidly respond to environmental stimuli within different domains of the cell. Neurons are highly polarized cells with cytoplasmic processes, axons and dendrites, that extend great distances from the cell body or soma. Several lines of evidence indicate that translation products of mRNAs localizing into axons of the peripheral nervous system (PNS) contribute to axon regeneration after injury (Smith et al., 2020). In rodents, PNS and some CNS axons extend centimeters from their soma, so this localized protein synthesis provides autonomy from the soma but also must be tightly regulated. Since one mRNA can be translated many times over to generate multiple copies of a protein, the survival of an mRNA within an axon can substantially affect the spatial and temporal regulation of that axon's proteome as well as its regeneration capacity (Dalla Costa et al., 2020). Much has been learned about how mRNAs are transported into and translated within axons over recent years, and it is clear that the axonal transcriptome is quite extensive in terms of numbers of different mRNAs and dynamic in terms of changes in mRNA populations with different physiological states (Kar et al., 2018). RNA binding proteins (RBP) and mRNAs assemble into a ribonucleoprotein particle (RNP) for transport into axons, and those or other RBPs can also subsequently regulate the translation of the mRNA in the axons or provide a storage depot to sequester the mRNA until needed (Dalla Costa et al., 2020). Both nonsense-mediated decay (NMD) and microRNA (miRNA)-stimulated RNA degradation have been shown to occur in distal axons (Aschrafi et al., 2012; Colak et al., 2013; Hengst et al., 2006; Murashov et al., 2007; Vargas et al., 2016). These observations point to an intrinsic capacity for locally depleting specific mRNAs from axons. RBP interactions have also been linked to mRNA stability, including axonally localized mRNAs. The neuronal protein HuD (also called ELAVL4) has been known to stabilize mRNAs by binding to AU-rich elements (AREs) in 3'UTRs of target mRNAs (Beckel-Mitchener et al., 2002). HuD protein localizes into distal neurites and has been implicated in transport and translation of some mRNAs (Bolognani et al., 
2004; Smith et al., 2004; Yoo et al., 2013). We previously showed that the KH splicing regulatory protein

(KHSRP; also known as KSRP, MARTA1, ZBP2, and FUBP2) decreases neurite growth in cultures of

embryonic cortical neurons and competes with HuD for binding to target mRNAs (Bird et al., 2013). In

contrast to HuD interactions, KHSRP binding destabilizes ARE-containing mRNAs (Gherzi et al., 2004).

Here, we show that KHSRP is one of several RBPs whose levels increase in PNS axons after injury and

during regeneration. This increase in axonal KHSRP occurs rapidly after PNS nerve injury through

translation of its mRNA in axons. Deletion of the KHSRP gene increases axonal levels of mRNAs encoding growth-associated proteins and this effect requires KHSRP's fourth hnRNP K homology (KH) domain that was shown to bind to ARE-containing mRNAs (Gherzi et al., 2004). Our data indicate that KHSRP slows axon growth through axon-intrinsic mechanisms, and point to autonomy of the axonal compartment for modulating levels of axonal mRNAs and, thereby, the axon's growth potential.

\section{RESULTS}

Peripheral nerve injury changes the axonal RNA binding protein population - Protein synthesis in PNS axons has been shown to facilitate nerve regeneration after injury. Transport and translation of the mRNA templates needed for this intra-axonal protein synthesis are driven by RBPs bound to those mRNAs (Dalla Costa et al., 2020). We recently showed that PNS axoplasm contains many RBPs that were thought to have exclusively nuclear roles by using RNA affinity mass spectrometry (RAMS) (Lee et al., 2018). The levels of some of these RBPs increased after axotomy, which pointed to functions in growing axons. As this RAMS assay focused on interactions with axonal mRNA localization motifs, we wanted to gain a more systematic and unbiased view of the axonal RBP population. With the limited amount of proteins obtained from PNS axoplasm coupled with the likely low abundance of RBPs relative to cytoskeletal components in the axons, we turned to a targeted mass spectrometry (MS) approach with parallel reaction monitoring (PRM) to profile axonal RBPs in naïve, injured and regenerating sciatic nerve axoplasm. By mining the UniProt database (https://www.uniprot.org) for proteins with 'RNA binding' in 
functional or domain descriptions and validated expression in the nervous system (cross-referencing each for RNA expression using the GeneCards database (https://www.genecards.org)), we arrived at 357 proteins to test. Of these, 196 were represented in a reference MS library that had been generated from rat nervous system tissues (including sciatic nerve, DRG, and spinal cord) and were included in a PRM-MS method that targeted 511 unique precursor peptides (Sahoo et al., 2018). Using this PRM method, we quantified the abundance of 84 RBPs represented by 184 precursor peptides in adult rat sciatic nerve axoplasm. Several of the proteins showed increases or decreases over 3-28 days post injury compared to the contralateral uninjured sciatic nerve (Figures 1A-B; Supplemental Figure S1). Initial validation by immunoblotting from naïve and 7 days post-crush injury sciatic nerve axoplasm showed that FXR1, hnRNP A3, hnRNP AB, hnRNP H1 and KHSRP increased in the 7 day samples (Figure 1C). Notably, hnRNP H1 was identified by the RAMS study for axonal RBPs binding to Nrn1 mRNA localization motif and we similarly found its axoplasm levels are increased after injury (Lee et al., 2018).

The increase in KHSRP after injury was surprising as the protein has been linked to mRNA destabilization in neurons, including targeting Gap43 mRNA for degradation whose encoded protein has long been linked to axon growth promotion (Bird et al., 2013). Examining KHSRP more closely, its levels increased in the sciatic nerve axoplasm by 3 days (Fig 1D-E). Moreover, the axoplasm KHSRP levels were significantly higher at 21-28 days post injury than earlier time points (Fig 1D-E). With the mid-thigh sciatic nerve crush used, rats here begin to regain lower hind limb function over 21-28 days post injury indicative of some target reinnervation. The axoplasm preparation used is highly enriched for axonal proteins, but does contain some glial constituents (Rishal et al., 2010). Since KHSRP is ubiquitously expressed (Li et al., 2012; Min et al., 1997), we used confocal microscopy to determine if the elevations in KHSRP levels seen by PRM and immunoblotting derived from axonal KHSRP. Consistent with the ubiquitous KHSRP expression, KHSRP protein immunofluorescence was seen in both axons and the adjacent Schwann cells; however, extraction of the KHSRP immunofluorescence overlapping with 
neurofilament signals, showed a markedly increased axonal KHSRP signals at 7 days post injury compared to uninjured nerve (Figure 1F). Thus, axotomy induces an early and lasting increase in axonal KHSRP levels.

\section{Loss of KHSRP enhances axon growth in the peripheral nervous system through an axon-intrinsic}

mechanism - With its role in targeting mRNAs for degradation and clear evidence indicating that axonal mRNA translation supports axon regeneration, we contemplated what function this increased axonal KHSRP might serve. To address this, we took advantage of a global KHSRP knockout mouse line, where both alleles of KHSRP are deleted (Li et al., 2012). Cultures of dissociated lumbar (L) 4-6 dorsal root ganglia (DRGs) neurons from $\mathrm{Khsp}^{-/-}$mice showed significantly increased axon growth compared to those from $\mathrm{Khsrp}^{+/+}$mice (Figure $2 \mathrm{~A}-\mathrm{B}$ ) but no differences in axon branching (Suppl. Figure S2A-B).

In vivo injury-conditioning by sciatic nerve crush injury has been shown to increase in vitro axon growth from dissociated DRG neurons independent of new gene transcription upon culturing (Smith and Skene, 1997). The in vivo conditioning injury activates transcription of growth-associated genes, whose mRNA products are then translationally regulated after the second injury that is brought by the DRG dissection and dissociation needed for culture (Twiss et al., 2000b). Thus, we tested whether axon growth from 'injury-conditioned' L4-6 DRG s is further increased in the absence of KHSRP. In contrast to the naïve DRG cultures, there was no significant difference in axon length comparing the injuryconditioned DRGs from $K h s r p^{-/}$vs. Khsrp ${ }^{+/+}$mice (Figure $2 \mathrm{~A}-\mathrm{B}$ ). To be certain that KHSRP was indeed absent from these neurons, we tested KHSRP levels in soma and axon lysates from L4-6 DRG neurons cultured neurons on a porous membrane for separation of axons (Willis and Twiss, 2011). KHSRP immunoreactive bands were clearly present in the $\mathrm{Khsrp}^{+/+}$somal and axonal lysates, but were not detected in the $\mathrm{Khsr}^{-\%}$ samples even with extended exposures (Figure $2 \mathrm{C}$ ). These data suggest that removing KHSRP can increase axon growth, but maximum growth promotion compared to $K h s r p^{+/+}$DRG cultures was only seen in naïve and not injury-conditioned neurons. 
With no differences in axon growth between in $\mathrm{Khsp}^{-/-}$and $K h s r p^{+/+}$injury-conditioned DRG neurons, this could suggest that the growth attenuating effects of KHSRP are overcome by the conditioning effects. However, since the MS data pointed to an increase of KHSRP within the axons of peripheral nerve after injury, an alternative hypothesis is that the growth-attenuating functions of KHSRP are axon-intrinsic. That is, shearing the DRG axons during in vitro culture preparation could negate any in vivo increase in KSHRP levels that is selective to the axons. To test this alternate hypothesis, we compared in vivo axon regeneration in the $K h s r p^{-/}$and $K h s r p^{+/+}$mice. There was a modest increase in axon regeneration in the sciatic nerve regeneration at 7 days after crush injury in

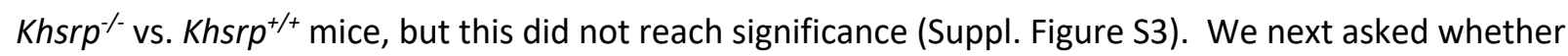
an in vivo injury-conditioning paradigm might fully distinguish the $K h s r p^{-/-}$and $K h s r p^{+/+}$mice, since the robust axotomy induced increase in axonal KHSRP would be absent in the $\mathrm{Khsrp}^{-/-}$mice. Thus, we performed a unilateral sciatic nerve crush injury and $7 \mathrm{~d}$ later performed a second ipsilateral crush proximal to the initial injury; the contralateral side underwent single crush at the same time as the second lesion (and same approximate level). $3 \mathrm{~d}$ later, nerve regeneration contralateral to the conditioning lesion (i.e., single crush injury) showed no significant difference between $\mathrm{Khsr}^{-/-}$and $K h s r p^{+/+}$mice (Figure 3A-B). In sharp contrast, the injury-conditioned $K h s r p^{-/}$nerves (i.e., double crush injury) showed a dramatic increase in axon regeneration compared to the injury-conditioned Khsrp ${ }^{+/+}$ mice (Figure $3 \mathrm{~A}-\mathrm{B}$ ). To determine if this increased axon growth accelerated target reinnervation, we analyzed neuromuscular junctions (NMJs) in the gastrocnemius muscle 14 days after crush injury in $\mathrm{Khsrp}^{+/+}$and $\mathrm{Khsrp}^{-/-}$mice (Figure 3C). NMJ occupancy based on percentage of presynaptic compared postsynaptic area showed no differences between $\mathrm{Khsr}^{+/+}$and $K h s r \mathrm{P}^{-/}$mice at 14 days after a single nerve crush (Figure 3D). However, the double crush injured mice showed significantly greater NMJ occupancy in $\mathrm{Khsr}^{-/-}$vs. $\mathrm{Khsrp}^{+/+}$(i.e., $14 \mathrm{~d}$ after the second crush lesion, $21 \mathrm{~d}$ after initial conditioning injury; Figure 3C-D). 
The differences seen above between in vivo nerve regeneration and in vitro axon growth could be consistent with axon-intrinsic effects of KHSRP. To test this possibility more directly, we developed an in vitro axotomy model where DRGs were initially allowed to extend axons and then the axon shafts were severed. This allowed us to assess regeneration in vitro from an injured axon rather than initiation of axon growth from the cell body reflected in the in vivo injury-conditioned DRG cultures used in Figure 2B above or replated DRG neurons used in other studies (Perry et al., 2016). DRG neurons from Khsrp ${ }^{-/}$ and $\mathrm{Khsr}^{+/+}$mice were cultured on membrane filters for $36 \mathrm{~h}$; the undersurface of the membrane was then scraped to sever the axons (Figure 3E). After an additional $72 \mathrm{~h}$ in culture, neurofilament immunostained axons were traced along the membrane undersurface and total axon lengths were quantified (Figure 3F). Consistent with increased regeneration from injury-conditioned nerves in vivo, the Khsrp/- DRGs showed significantly accelerated axon regeneration after their axon shafts were severed in vitro (Figure 3G). Taken together, these data indicate that the intra-axonal increase in KHSRP following axotomy actually slows growth of the regenerating axon.

The axotomy-induced increase in axonal KHSRP occurs via axon-intrinsic translation - Translation of mRNA in axons provides a means to rapidly change protein content in subcellular domains. For example, injury-induced translation of axonal Calr mRNA was recently shown to support early regrowth of severed axons (Pacheco et al., 2020). Thus, we wondered whether the change in axonal KHSRP levels might be driven by localized translation of its mRNA. We had previously detected Khsrp mRNA in RNAseq analyses of sciatic nerve axoplasm (Lee et al., 2018); however, these axoplasm preparations obviously can contain non-neuronal contents (Rishal et al., 2010), particularly for mRNAs isolated from injured nerve as Lee et al. (2018) used. To overcome this limitation, we used single molecule fluorescence in situ hybridization (smFISH) combined with IF to ask whether sensory axons contain Khsrp mRNA. Dissociated DRG cultures from $\mathrm{Khsrp}^{+/+}$mice showed prominent $K h s r p$ mRNA signals in cell body and axons (Figure 4A-C). The axonal RNA signal was granular (Figure 4A), as typically seen for axonal and 
dendritic mRNAs. There was no Khsrp mRNA signal in the axons of the Khsrp ${ }^{-/}$DRG cultures (Figure 4C). Similarly, smFISH/IF performed on sciatic nerve sections showed prominent Khsrp mRNA signal in the

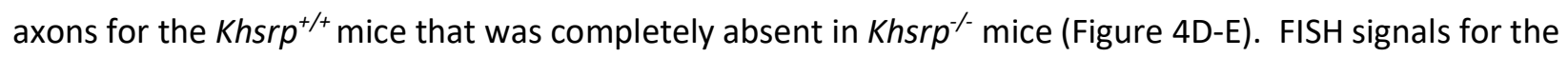
cell bodies of the DRGs from $K h s r{ }^{-/}$mice did show a faint but consistent signal with the Khsrp mRNA probes in the cell bodies that was significantly greater than scrambled probe (Figure 4A-B). The Khsrp ${ }^{-/}$ mice were generated by deleting exons 1-13 (of 18) of the murine KHSRP gene (Lin et al., 2011), and the Stellaris smFISH Khsrp mRNA probes used here hybridize to sequences across the full exons comprising the mature Khsrp mRNA. Thus, with absence of KHSRP protein in these cultures based on Figure 2C, we suspect that this is an RNA transcribed from exons 14-18 remaining in the Khsrp gene, or portions thereof as confirmed by RNA-sequencing of $\mathrm{Khsrp}^{-/}$adult mouse brain and embryonic cortical neuron cultures (data not shown). Nonetheless, the strong Khsrp mRNA signal in the axons of cultured DRG neurons and in vivo sciatic nerve indicate that Khsrp mRNA localizes into PNS axons.

The RNA analyses above suggest that KHSRP may be synthesized locally in axons. To determine if the PNS nerve injury-induced increase in KHSRP is intrinsic to the nerve rather than transported from cell body, we ligated the sciatic nerve to restrict anterograde transport and performed a crush injury distal to the ligation (Figure 5A-B). Adult rats were used for these ligation analyses since the larger sciatic nerve could be ligated more consistently than in the mouse. Immunofluorescence for amyloid precursor protein (APP) and Signal transducer and activator of transcription $3 \alpha$ (Stat3 $\alpha$ ) confirmed that the ligation attenuated both anterograde and retrograde transport, as APP accumulated proximal to and Stat $3 \alpha$ accumulated distal to the ligation site (Suppl. Figure S4A). As expected KHSRP signals were detected both in axons and adjacent Schwann cells (Figure 5B-C, Suppl. Figure 4B-C). Axonal KHSRP Immunofluorescence showed increased signals at both the ligation (proximal and distal) as well as at the crush site relative to axons in naïve nerve; however, the increased axonal KHSRP signals proximal to the crush site were significantly greater and continued to elevate over time after injury compared to the 
ligation sites (Figure 5B-C, Suppl. Figure 4B-C). These results are consistent with local synthesis of KHSRP in the injured nerve.

To more directly test if KHSRP is translated in the sciatic nerve axons, we exploited an ex vivo nerve injury model where we used puromycin incorporation to detect nascent peptides from the axoplasm extruded from the nerve segments (Terenzio et al., 2018). We excised segments of rat sciatic nerve and placed these into culture medium; cyclosporin A was included to delay Wallerian degeneration (Barrientos et al., 2011) and O-parpargyl-puromycin (OPP) was included for puromycinylation of nascently synthesized polypeptides (Forester et al., 2018; Sahoo et al., 2020). This ex vivo assay showed a significant increase in puromycinylated KHSRP in the axoplasm isolates at $4 \mathrm{~h}$ after nerve crush that was attenuated by the protein synthesis inhibitor anisomycin (Figure 5D-E). Notably, an increase in overall KHSRP levels was seen in the crushed nerve segment axoplasm and this was also attenuated by pretreatment with anisomycin (Figure 5D), confirming that axonal injury increases axonal KHSRP levels through mRNA translation. To further test for translation of Khsrp mRNA in axons, we fused 5' and 3'UTRs of rodent Khsrp mRNA to the coding sequence of a diffusion-limited GFP reporter CDNA (GFPMYR5'/3'khsrp; Figure 5F) to use as a surrogate for axonal localization and translation of Khsrp mRNA. By fluorescence recovery after photobleaching (FRAP), DRG neurons expressing GFPMYR $5^{\prime} / 3^{\prime}$ khsrp showed rapid fluorescent recovery that was significantly attenuated by pretreatment with anisomycin or a second protein synthesis inhibitor cycloheximide (Figure 5F; Suppl. Figure S5). Taken together these results indicate that the axotomy-induced increase in axonal KHRP is derived from localized translation of Khsrp mRNA in axons.

KHSRP's ARE-binding KH4 domain attenuates axon growth - KHSRP was previously shown to bind to the ARE in Gap43 mRNA's 3'UTR and destabilize the transcript (Bird et al., 2013). The GAP43 gene is transcriptionally activated following PNS nerve injury (Van der Zee et al., 1989), and axonal Gap43 mRNA levels increase more than 2 fold in regenerating PNS axons (Yoo et al., 2013). Given that Gap43 
mRNA and protein expression are typically associated with axon growth (Skene, 1989), we tested the effect of Khsrp knockout on the sciatic nerve levels of this KHSRP target mRNA. RTddPCR from sciatic nerve samples of $\mathrm{Khsr}^{+/+}$mice showed the expected increase in Gap43 mRNA at 7 days after nerve crush injury (Figure 6A). However, the sciatic nerve levels of Gap43 mRNA in Khsrp ${ }^{-1-}$ mice were approximately 2-fold higher than in $\mathrm{Khsr}^{+/+}$mice after crush injury (Figure 6A). Gap43 mRNA levels in injured $K h s r p^{+/-}$mouse nerves were intermediate between $K h s r p^{+/+}$and $K h s r p^{-/-}$nerves, but this did not reach statistical significance. There were only modest, non-significant increases in nerve Gap43 mRNA for uninjured $K h s r p^{-/-}$and $K h s r p^{+/}$vs. Khsrp ${ }^{+/+}$nerves (Figure 6A). Since KHSRP can potentially bind to many different axonal mRNAs, we asked if axonal Snap25 mRNA might be affected by Khsrp knockout. We recently found that Snap25 mRNA is a target for destabilization by KHSRP in mouse brain(Olguin et al., 2020), and the Hengst lab had shown that translation of Snap25 mRNA in CNS axons promotes synaptogenesis (Batista et al., 2017). Snap25 mRNA was detected in sciatic nerve, and its levels were significantly increased in the injured sciatic nerves of $\mathrm{Khsp}^{-/-}$compared to $\mathrm{Khsr}^{+/+}$mice (Figure $6 \mathrm{~B}$ ). In contrast, there was no difference in the levels of Hmgb1 mRNA (also called Amphoterin; Figure 6C), an mRNA that we have previously shown localizes to axons (Merianda et al., 2015) but was not affected by Khsrp knockout nor did it bind to KHSRP in RIP-Seq analyses (Olguin et al., 2020). Thus, increased levels of mRNAs encoding proteins linked to axon growth accompany the increased axon regeneration seen in injury-conditioned $\mathrm{KhsP}^{-/-}$mice.

Previous work has shown that the $\mathrm{KH} 3$ and $\mathrm{KH} 4$ domains of KHSRP are needed for ARE binding and mRNA degradation via the cytoplasmic exosome complex (Gherzi et al., 2004), and deletion of KH4 attenuates the effects of KHSRP on neurite growth in embryonic CNS neuron cultures (Bird et al., 2013). To determine if KHSRP regions previously linked to mRNA decay determine the axonal growthattenuation by KHSRP in adult neurons, we transfected $K h s r p^{+/+}$and $K h s r p^{-/}$DRG cultures with full length GFP-KHSRP or GFP-KHSRP with KH4 domain deleted (GFP-KHSRPAKH4). GFP-KHSRP reversed the axon 
growth promoting effects of KHSRP loss in the Khsrp ${ }^{-/-}$DRGs and decreased axon growth in $\mathrm{Khsr}^{+/+}$DRGs

(Figure 6D-E). In contrast, GFP-KHSRPAKH4 expression only modestly decreased axon growth in the $K h s r p^{-/}$DRGs and had no significant effect on $K h s r p^{+/+}$DRGs (Figure 6D-E). Axonal and cell body Gap43 mRNA levels showed comparable results, with GFP-KHSRP expression significantly decreasing Gap43 mRNA levels in $\mathrm{Khsrp}^{+/+}$and $\mathrm{Khsrp}^{-/}$DRGs, while only modest or no decreases were seen with GFP-

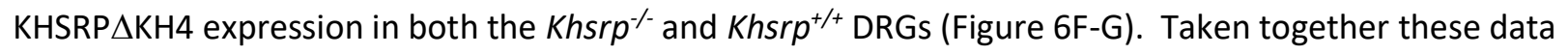
indicate that the RNA degradation activity of KHSRP determines its ability to slow axon growth and decrease axonal levels of mRNAs encoding growth-associated proteins.

\section{DISCUSSION}

RBPs play critical roles in determining localized neuronal protein levels through posttranscriptional control mechanisms. Since one mRNA can generate multiple copies of protein, RNA stability needs to be tightly regulated. HuD and KHSRP both bind to ARE-containing mRNAs, with HuD stabilizing and KHSRP destabilizing the bound mRNAs. We show that PNS axons contain many different RBPs whose levels change after injury, including KHSRP. Axonal KHSRP protein levels rapidly increase in peripheral nerves following axotomy through translation of axonal Khsrp mRNA. This increase in axonal KHSRP likely destabilizes axonal mRNAs as Khsrp ${ }^{-/}$mice show increased axonal levels of Gap43 and Snap25 mRNAs, two KHSRP target mRNAs, in injured sciatic nerves compared to $\mathrm{Khsrp}^{+/+}$mice. Moreover, deletion of the Khsrp gene accelerates regeneration of severed axons both in vitro and in vivo, indicating that intra-axonal translation of Khsrp mRNA slows axon regeneration. Since this effects axon regeneration from a severed axon rather than initiation of axon growth from the cell body, our results indicate that KHSRP's role in axon growth is through its localized function in the axon. Together these data point to injury-induced increase in axonal KHSRP as an axon-intrinsic determinant of regeneration capacity. 
KHSRP has been implicated in RNA splicing, trafficking and degradation as well as microRNA biogenesis (Gherzi et al., 2004; Min et al., 1997; Pan et al., 2007; Trabucchi et al., 2009). Our analyses of KHSRP target mRNA levels in sciatic nerves of the $\mathrm{Khsp}^{-/}$-mice point to KHSRP's role in RNA degradation and resulting acceleration of axon growth seen in these mice. miRNAs are clearly linked to stability of mRNAs, and their precursors (pre-miRNAs) have been shown to localize into PNS axons, with PNS nerve injury triggering localized processing of some axonal pre-miRNAs into mature miRNAs (Kim et al., 2015). However, levels of previously reported KHSRP target microRNAs (Trabucchi et al., 2009) showed no changes when comparing DRG cultures from $K h s r p^{-/}$vs. $K h s r p^{+/+}$mice (data not shown). Transfecting the Khsrp ${ }^{-/}$DRG neurons with wild type vs. KH domain mutant KHSRP expression constructs further point to KHSRP-mediated RNA degradation rather than a secondary miRNA-mediated effect for KHSRP's role in axon growth. KHSRP has four $\mathrm{KH}$ domains of about 70 amino acid residues each that can bind to single strand DNA or RNA with varying degree of specificity (Gherzi et al., 2004). The third and fourth KH domains ( $\mathrm{KH} 3$ and $\mathrm{KH} 4$ ) are implicated in RNA decay by binding to ARE-containing mRNAs and recruiting components of the cytoplasmic exosome complex used for RNA degradation (Gherzi et al., 2004). We find both the axon growth promotion and increase in axonal Gap43 mRNA in adult DRG neurons require an intact $\mathrm{KH} 4$ domain that has been shown to bind to ARE-containing mRNAs, arguing for a direct effect of KHSRP on stability of the axonal mRNAs rather than indirectly through microRNAs or other mediators. Our recent RNA-seq analyses of brain from $\mathrm{Khsr}^{-/-}$vs. $\mathrm{Khsr}^{+/+}$mice and mRNAs co-immunoprecipitating with KHSRP from wild type brain uncovered 527 KHSRP target mRNAs (Olguin et al., 2020). Thus, KHSRP likely binds to many other axonal mRNAs beyond Gap43 and Snap25 to regulate their stability. However, we cannot completely exclude indirect effects from KHSRP deletion used here, and consistent with this possibility, the work from Olguin et al. (2020) showed evidence for this with 1400 mRNAs that decreased in brains of $\mathrm{Khsp}^{-/}$but showing no binding to KHSRP in RIP assays. 
Several lines of evidence indicate that mRNAs can compete for interactions with RBPs and different RBPs can compete with one another for interactions with mRNAs. KHSRP as well as Gap43 mRNA have been implicated in these competitions. We have previously shown that localized translation of Gap43 mRNA contributes to elongating growth of DRG axons (Donnelly et al., 2013). ZBP1, in complex with HuD, is needed for axonal localization of Gap43 mRNA (Yoo et al., 2013), and ZBP1 is also needed for axonal localization of Actb mRNA (Zhang et al., 2001). Interestingly, ZBP1 is expressed at limiting levels in adult DRG neurons such that Actb and Gap43 mRNAs compete for localization into axons (Donnelly et al., 2013; Donnelly et al., 2011), with the axotomy-induced transcriptional increase in Gap43 mRNA altering the stoichiometry of neuronal Actb to Gap43 mRNA, and decreasing axonal localization of Actb mRNA (Yoo et al., 2013). Nrn1 and Gap43 mRNAs compete for binding to HuD with Nrn1 mRNA's ARE showing a higher binding affinity for HuD than Gap43 mRNA's ARE (Gomes et al., 2017). Furthermore, HuD and KHSRP compete for binding to Gap43 mRNA, but they bind to the mRNA with relatively the same affinity (Bird et al., 2013). Consequently, the injury-induced increase in axonal KHSRP levels that we see here would effectively alter the localized stoichiometry of HuD to KHSRP, thereby favoring KHSRP binding to axonal Gap43 mRNA and its subsequent degradation. Consistent with this prediction, previous RAMS analyses for proteins in crushed sciatic nerve axoplasm binding to Gap43 mRNA's ARE (nt 1211-1250) detected KHSRP and related KH-domain containing FUBP1 and FUBP3, but not HuD (Lee et al., 2018).

It is intriguing to speculate that the increase in Snap25 mRNA, which was also elevated in the axoplasm of $\mathrm{Khsp}^{-/-}$mice, is similarly affected by this competition. Snap25 mRNA contains two AREs in the 3 'UTR at nt 1085-1102 and 1586-1598 (Genbank \# NM_001355254.1) that could bind to both KHSRP and HuD (Olguin et al., 2020). SNAP25 is a cytoplasmic SNARE protein involved in neurotransmitter release (Sollner et al., 1993), but SNAP25 also has reported roles in both chick retina and cortical axon development (Osen-Sand et al., 1993). Axonal translation of Snap25 mRNA has been linked to synapse 
development in cortical neurons (Batista et al., 2017), so elevated axonal Snap25 mRNA in combination with Gap43 mRNA could contribute to the accelerated NMJ occupancy shown here in the Khsrp ${ }^{-1}$ compared to $\mathrm{Khsp}^{+/+}$mice after conditioning crush lesions. With both Gap43 and Snap25 mRNAs linked to positive outcomes for axon growth and regeneration, these data raise the possibility that the increase in axonally synthesized KHSRP after axonal injury likely slows axon growth by decreasing the survival of mRNAs that constitute an RNA regulon supporting axon growth.

The initial increase in axonal KHSRP protein through translational upregulation of its axonal mRNA shows similar kinetics to translational induction of injury-associated mRNAs following axotomy. mRNAs encoding Importin $\beta 1$ (Kpnb1 mRNA), Vimentin (Vim mRNA), RanBP1, Stat3, mTor and Calreticulin (Calr mRNA) are translated in axons within the first six hours following PNS nerve crush injury (Ben-Yaakov et al., 2012; Hanz et al., 2003; Perlson et al., 2005; Terenzio et al., 2018; Yudin et al., 2008). Some of those locally synthesized injury-associated proteins help to signal retrogradely to the soma for changing gene expression, including transport of transcription factors, to support the injured neuron's survival and axon regeneration (Rishal and Fainzilber, 2014). A retrograde calcium wave has also been shown to support axon regeneration by altering neuronal gene expression after PNS injury through epigenetic mechanisms (Cho et al., 2013). Together, these changes in gene expression likely contribute to the enhanced regeneration seen in injury-conditioned neurons. Our results showing that localized translation of Khsrp mRNA slows axon regeneration in injury-conditioned neurons, emphasize two critical points for the mechanisms underlying the injury-conditioning effect. First, there clearly is a response to injury conditioning that is limited to the axon since $K h s \mathrm{p}^{-/-}$injury-conditioned neurons only show enhanced axon regeneration when growth is initiated from an existing axon rather than new axon growth from the cell body. Second, this axon-intrinsic injury-conditioning effect slows axon growth, so it locally offsets the soma response to nerve injury by lowering levels of axonal mRNAs encoding growthassociated proteins. Taken together, our observations indicate that the axon growth rate seen from 
wild type injury-conditioned neurons is not the maximum attainable growth. However, slowing axon regeneration may be beneficial at some stages of recovery from PNS injury such as when axons reach target tissues. It is intriguing to speculate that the increase in axonal KHSRP levels as the adult animals used here begin to recover lower hind limb function after sciatic nerve crush injury may reflect such a need for axonal KHSRP.

Notably, the targeted proteomics approach used here uncovered 84 RBPs in sciatic nerve axoplasm, with many beyond KHSRP showing increased or decreased levels after nerve injury and during regeneration. Since the axoplasm isolates used here rely on extrusion in detergent-free conditions, we likely miss many RBPs that are associated with cytoskeleton or axoplasmic membrane. HuD/ELAVL4 has previously been reported to fractionate with the cytoskeleton in rat hippocampus (Pascale et al., 2004). So this may explain the decrease in HUD/ELAVL4 detected in the sciatic nerve axoplasm after injury by MS shown in Figure 1 above compared to our previous immunofluorescent analyses shown that axonal HuD/ELAVL4 levels increase during regeneration (Yoo et al., 2013). Nonetheless, considering the limitations in starting materials and sensitivity for protein detection compared to RNAs that can be amplified, we substantially increase the number of known axonal RBPs with the PRM approach. Moreover, we have uncovered a localized role for KHSRP in slowing PNS axon regeneration that is precipitated by localized translation of Khsrp mRNA in axons after injury. Our results expand the known functions for axonal RBPs and intra-axonal mRNA translation, and emphasize the potential to increase axon regeneration rates beyond the $1-2 \mathrm{~mm} / \mathrm{d}$ typically seen after PNS injury.

\section{MATERIALS AND METHODS}

Animal use and survival surgery - the Institutional Animal Care and Use Committee of the University of South Carolina approved all animal procedures. Adult male Sprague Dawley rats (175-250 g) or both male and female Khsrp knockout (Khsrp ${ }^{-/}$) (Lin et al., 2011) and wild type $\left(K h s r p^{+/+}\right.$) mice on C57/BI6 background were used for experiments. Wild type animals were typically littermates, and heterozygous 
animals $\left(\mathrm{Khsr}^{+/-}\right)$were used in several experiments as indicated. Isoflurane inhalation was used for anesthesia in all survival surgery experiments (see below). Animals were euthanized by $\mathrm{CO}_{2}$ asphyxiation at 3-7 d post-injury as indicated in results.

For peripheral nerve injury, anesthetized male rats or mice were subjected to sciatic nerve crush at mid-thigh level as previously described (Twiss et al., 2000a). For 'double crush 'injury experiments, a unilateral peripheral sciatic nerve crush was then performed at mid-thigh level on day 0 , as a 'conditioning lesion', and a second crush injury was performed at $0.5 \mathrm{~cm}$ proximal to the first crush site. Sciatic nerve ligations were performed in male rats as described previously as the larger size of these animals provided greater precision in ligation and subsequent crush injuries (Cavalli et al., 2005). Briefly, rat sciatic nerve was ligated approximately $1 \mathrm{~cm}$ proximal to planned mid-thigh nerve crush site. Immediately after applying 4.0 suture, the sciatic nerve was crushed distal to the ligation site as above and then euthanized 3-16 hours later.

Cell Culture - Dissociated cultures of adult DRGs were prepared as described (Twiss et al., 2000a). For experiments with naïve DRG neurons, all lumbar, thoracic, and lower cervical DRGs were collected. To study effects of an in vivo injury conditioning, L4-6 DRGs were used from ipsi-lateral (injury-conditioned) or contra-lateral (naïve) to the crush injury. DRGs were harvested in Hybernate-A medium (BrainBits, Springfield, IL) and then dissociated with collagenase as described. After centrifugation and three washes in DMEM/F12 (Life Technologies, Grand Island, NY), dissociated ganglia were cultured in complete medium containing DMEM/F12, 1 x N1 supplement (Sigma, St. Louis, MO), $10 \%$ fetal bovine serum (Hyclone, Logan, UT), and $10 \mu \mathrm{M}$ cytosine arabinoside (Sigma) on poly-L-lysine (Sigma) plus laminin (Millipore, Burlington, MA) coated substrates. For imaging, dissociated DRGs were cultured on coated glass coverslips. For analyses of axonal RNA levels or in vitro regeneration assay (see below), dissociated ganglia were cultured polyethylene-tetrathalate (PET) membrane inserts (1 $\mu \mathrm{m}$ pores; 
Falcon-Corning, Tewksbury, MA) (Zheng et al., 2001). Axons and CB were isolated from DRGs cultured on PET membranes as described (Willis et al., 2007).

For transfection, dissociated ganglia were pelleted at $100 \times \mathrm{g}$ for $5 \mathrm{~min}$ and resuspended in 100 $\mu \mathrm{l}$ 'Nucleofector solution '(Rat Neuron Nucleofector kit; Lonza, Alpharetta, GA). 4-6 $\mu \mathrm{g}$ of plasmid was electroporated using the AMAXA Nucleofector device (Neurons Rat DRG, G-013 program; Lonza) before plating and maintained for $48 \mathrm{~h}$.

Plasmid constructs - pAc-GFP-KHSRP and pAc-GFP-KHSRPAKH4 constructs have been published (Bird et al., 2013). All fluorescent reporter constructs for analyses of RNA translation were based on eGFP with myristoylation element (GFPMY; originally provided by Dr. Erin Schuman, Max-Plank Inst., Frankfurt) (Aakalu et al., 2001). cDNAs for the 5'UTR and 3'UTRs of Khsrp mRNA were custom synthesized by Integrated DNA Technologies ([IDT] Coralville, IA) and GenScript Biotech (Piscataway, NJ), respectively. The 5'UTR was engineered with 5 'Nhe1 and 3 'BamH1 restriction sites and cloned into pGFP MYR $5^{\prime}$ camk2a/3'actg (Willis et al., 2007), replacing the 5'UTR of CamK2a [GFP ${ }^{\text {MYR }} 5^{\prime}$ khsrp/3'actg]. The 3'UTR sequence was engineered with 5 'Not1 and 3 'Xho1 restriction sites and used to replace the Actg 3'UTR in pGFP ${ }^{M Y R} 5^{\prime}$ khsrp/3'actg plasmid [pGFP MYR 5 '/3'khsrp].

PRM-MS quantitation of RBPs - Axoplasm from $2 \mathrm{~cm}$ segments of sciatic nerve immediately proximal to crush site was extruded into nuclear transport buffer (20 mM HEPES [pH 7.3], 110 mM potassium acetate, $5 \mathrm{mM}$ magnesium acetate) supplemented with protease/phosphatase inhibitor cocktail (Roche) and RNasin Plus (Promega, Madison, WI). Contralateral (uninjured) sciatic nerve of comparable level and length was used for control. 3 animals were used for each time point and both naïve and injured sciatic nerve axoplasm. Preparations were cleared by centrifugation at $20,000 \times \mathrm{g}, 4^{\circ} \mathrm{C}$ for $30 \mathrm{~min}$, supernatants were diluted in $0.5 \mathrm{ml}$ of Trlzol LS reagent (Invitrogen) and protein was extracted according to the manufacturer's protocol. Protein pellets were digested with trypsin as previously described (Urisman et al., 2017). 
PRM was performed on Q Exactive Plus Mass Spectrometer (Thermo-Fisher) online with nanoAcquity UPLC System (Waters, Milford, MA). Digested peptides samples $(0.5 \mu \mathrm{g})$ were injected onto $200 \mathrm{~cm}$ monolithic silica-C18 column (GL Sciences, Tokyo, Japan) and separated using a 6 hour reversed phase chromatography gradient as previously described (Urisman et al., 2017). The mass spectrometer was operated in PRM mode with the following parameters: positive polarity, $R=17,500$ at $200 \mathrm{~m} / \mathrm{z}, \mathrm{AGC}$ target 1e6, maximum IT 190 ms, MSX count 1, isolation window 3.0 m/z, NCE $35 \%$. PRM data were analyzed in Skyline v. 3.5 (MacLean et al., 2010). Skyline PRM document has been uploaded to PanoramaWeb Public (Sharma et al., 2018) and can be accessed at https://panoramaweb.org/axonrbps.url.

RNA isolation and PCR analyses - RNA was isolated from dissociated DRG neurons or cell body/axon compartments collected from insert cultures using RNeasy Microisolation kit (Qiagen). Sciatic nerve was cut in small pieces and digested with collagenase at $37^{\circ} \mathrm{C}$ for 30 min with intermittent trituration. RNA was isolated from the collagenase-treated nerve using Trizol LS reagent (Invitrogen, Carlsbad CA) according to manufacturer's instructions. RNA concentration was measured by fluorimetry with Ribogreen (Life Technologies) and 10-50 ng of RNA was reverse transcribed with Sensifast cDNA synthesis kit (Bioline, London, UK). DRG axonal purity was assessed by RT-PCR, performed with primers designed to detect cell body-restricted mRNAs (cJun and Map2) and glial cell-specific mRNAs (Gfap). ddPCR was performed according to manufacturer's procedure with Evagreen detection (Biorad, Hercules, CA). Mitochondrial 12S ribosomal RNA (Mtrnr1) and Hmgb1 mRNA levels were used for normalizing RNA yields across different isolates. Following primers were used RT-PCR and RTddPCR (all from IDT, listed as 5 'to 3'): Mtrnr1, sense - GGCTACACCTTGACCTAACG and antisense CCTTACCCCTTCTCGCTAATTC; Actb, sense - CTGTCCCTGTATGCCTCTG and antisense ATGTCACGCACGATTTCC; cJun, sense - GCAAAGATGGAAACGACCTTCTAC and antisense AAGCGTGTTCTGGCTATGC Gfap, sense - AGTTACCAGGAGGCACTTG and antisense - 
GGTGATGCGGTTTTCTTCG; Hmgb1, sense - CATGGGCAAAGGAGATCC and antisense -

CTCTGAGCACTTCTTGGAG; Gap43, sense - CAGGAAAGATCCCAAGTCCA and antisense -

GAGGAAAGTGGACTCCCACA; Map2, sense - CTGGACATCAGCCTCACTCA and antisense -

AATAGGTGCCCTGTGACCTG; and, Snap25, sense - CAAATTTAACCACTTCCCAGCA and antisense -

\section{CAGAATCGCCAGATCGACAG.}

Immunofluorescent staining - Immunofluorescence was performed as previously described with all

steps at room temperatures unless specified otherwise. Coverslips were fixed with $4 \%$

paraformaldehyde (PFA) in phosphate-buffered saline (PBS) for $15 \mathrm{~min}$ at room temperature and

washed 3 times in PBS. PBS washed neurons were permeabilized with $0.3 \%$ Triton X-100 in PBS for 15

min and then blocked in $5 \%$ BSA for $1 \mathrm{~h}$. Neurons were incubated with primary antibodies overnight in

humidified chambers at $4^{\circ} \mathrm{C}$. Primary antibodies consisted of: chicken anti-NFH, NFM and NFL cocktail

(1: 500; Aves Lab, NFH \# AB_2313552, NFM \# AB_2313554, and NFL \# AB_2313553), RT97 mouse anti-

NF (1:500; Devel. Studies Hybridoma Bank, lowa City, IA). After washes in PBST, coverslips were

incubated with combination of FITC-conjugated donkey anti-mouse, Cy5 conjugated donkey anti-chicken

(both at 1:500; Jackson ImmunoRes., West Grove, PA) as secondary antibodies for $1 \mathrm{~h}$. After $1 \mathrm{~h}$,

coverslips were washed 3 times in PBS, rinsed with distilled $\mathrm{H}_{2} \mathrm{O}$, and mounted with Prolong Gold

Antifade with DAPI (Life Technologies).

For regeneration studies on mouse sciatic nerve and quantifying axonal content of KHSRP in

vivo, sciatic nerve segments were fixed for $4 \mathrm{~h}$ in $4 \%$ PFA and then cryoprotected overnight in $30 \%$

sucrose in PBS at $4^{\circ} \mathrm{C} .10 \mu \mathrm{m}$ cryostat sections for rat sciatic nerve and $20 \mu \mathrm{m}$ cryostat sections for

mouse sciatic nerve were processed for immunostaining as previously described (Merianda et al., 2013).

Primary antibodies consisted of RT97 mouse anti-NF (1:500), rabbit anti-KHSRP (1:200; Novus

Biologicals, NBP1-8910, Centennial CO), rabbit anti-Stathmin-2/SCG10 (1:500; Novus Biologicals, NBP1-

49461). Stathmin-2/SCG10 immunofluorescence was used to detect regenerating mouse sciatic nerve 
axons (Shin et al., 2012). Cy3-conjugated donkey anti-rabbit and FITC-conjugated donkey anti-mouse in combination were used as secondary antibodies for rat sciatic nerve (both at 1:500, Jackson ImmunoRes). Cy3-conjugated donkey anti-rabbit antibodies were used on mice sciatic nerve (1:500, Jackson ImmunoRes).

All samples were mounted with Prolong Gold Antifade and imaging was performed at room temperature. Samples were analyzed by either epifluorescent or confocal microscopy. Leica DMI6000 epifluorescent microscope with ORCA Flash ER CCD camera (Hamamatsu) was used for epifluorescent imaging. Confocal imaging for immunofluorescence was performed on a Leica SP8X microscope (DMI6000 M platform; Buffalo Grove, IL) fitted with a galvanometer Z stage and HyD detectors; HC PL Apo 63x/1.4 NA objective (oil immersion) was used with acquisition parameters matched for individual experiments using LAS-X software. Z-stack images were post-processed by Leica Lightning Deconvolution integrated into LASX software. Deconvolved image stacks were projected into single plane images using the maximum pixel intensities.

Neuromuscular Junction staining - NMJ staining protocol was performed as previously published with minor modifications (Maimon et al., 2018). Briefly, all steps were carried out at room temperature. Gastrocnemius muscle (GM) was cleared of any connective tissue, washed in PBS, fixed in $4 \%$ PFA, washed in PBS 3 times for 5 min each. Muscle was then dissected into smaller pieces and incubated with $1 \mu \mathrm{g} / \mathrm{ml}$ of Alexa Fluor 488 conjugated $\alpha$-Bungarotoxin for $4 \mathrm{~h}$ with rocking (Thermo-Fisher, B13422). Tissues were washed with PBS 3 times for 5 min each, treated with methanol at $-20^{\circ} \mathrm{C}$ for 5 min, and rinsed in PBS 3 times for 5 min each with rocking. Tissues were blocked for $1 \mathrm{~h}$ with $2 \%$ BSA, $0.4 \%$ Triton X-100 in PBS. Tissues were then incubated overnight with the following cocktail of primary antibodies to presynaptic components diluted in blocking solution with rocking: rabbit anti-NF 200 (1:200; Sigma, N4142), mouse anti-synaptophysin (1:300; Millipore, MAB5258), rabbit anti-synapsin-I, (1:200; Millipore, AB1543P). The following day, tissues were rinsed 3 times 5 min each in PBS while 
rocking. Samples were then incubated in Cy3-conjugated donkey anti-rabbit and Cy3-conjugated donkey anti-mouse antibodies (1:500; Jackson ImmunoRes) for $4 \mathrm{~h}$. After rinsing in PBS, muscle fibers were spread into monolayers under a stereomicroscope and affixed to slides using Prolong Gold Antifade; coverslips were sealed with clear nail polish.

Leica SP8X microscope as above was used for imaging. Sequential scanning was used to separate the green and red channels and Z stack with $200 \mathrm{~nm}$ interval using a HC PL Apo 63x/1.4 NA oil immersion objective.

Fluorescence in-situ hybridization (FISH) - smFISH plus IF was used to detect Khsrp mRNA in DRG and sciatic nerve. We used custom designed Cy3-labelled Stellaris probes (LGC Biosearch Tech, Middlesex, UK) for mouse Khsrp mRNA (Genbank ID \# NM_010613.3) with Cy3-labelled scramble probe for control. RT97 mouse anti-NF (1:200) was used as a primary antibody and FITC-conjugated donkey anti-mouse (1:200) was used as secondary. Samples processed without addition of primary antibody served as control for antibody specificity. Samples were mounted with Prolong anti-fade.

smFISH/IF on DRG cultures was performed as described previously (Kalinski et al., 2015). Briefly, coverslips were rinsed in PBS and then fixed in buffered $2 \%$ paraformaldehyde for $15 \mathrm{~min}$, with all steps carried out at room temperature unless specified otherwise. Coverslips were rinsed 2 times in PBS, then permeabilized in $0.3 \%$ Triton X-100 in PBS for 5 min. Samples were equilibrated for $5 \mathrm{~min}$ in hybridization buffer ( $50 \%$ dextran sulphate, $10 \mu \mathrm{g} / \mathrm{ml}$ E. coli tRNA, $10 \mathrm{mM}$ ribonucleoside vanadyl complex, $80 \mu \mathrm{g} \mathrm{BSA}$, and $10 \%$ formamide in $2 \mathrm{XSSC}$ ), and then incubated with $12.5 \mu \mathrm{M}$ probe plus mouse anti-NF (1:200) for $12 \mathrm{~h}$ at $37^{\circ} \mathrm{C}$. Coverslips were then washed in PBS + 0.3 \% Triton X-100 3 times, followed by incubation with FITC-conjugated donkey anti-mouse for $1 \mathrm{~h}$. After rinse in PBS, post fixation in $2 \%$ PFA for 15 min, and a second PBS wash, coverslips were inverted and mounted on glass slides. 
Detection of mRNA in mouse tissues was done as previously described (Kalinski et al., 2015), with all steps carried out at room temperature unless specified otherwise. Briefly, sciatic nerve segments were fixed for $4 \mathrm{~h}$ in $2 \%$ PFA, cryoprotected overnight in $30 \%$ buffered sucrose at $4^{\circ} \mathrm{C}$, and then cryosectioned at $20 \mu \mathrm{m}$ thickness (sections were stored at $-20^{\circ} \mathrm{C}$ until used). Sections were brought to room temperature, washed three times in PBS for 5 min each, and then treated with $20 \mathrm{mM}$ glycine and fresh $0.25 \mathrm{M} \mathrm{NaBH}_{4}$ in PBS (3 times, 10 min each for both) to quench autofluorescence. Sections were quickly rinsed in $0.1 \mathrm{M}$ Triethylamine (TEA) and then incubated in $0.1 \mathrm{M}$ TEA $+0.25 \%$ Acetic Anhydride for 10 min. Sections were dehydrated in 70, 95, and $100 \%$ ethanol ( 3 min each) and then delipidated in chloroform for 5 min followed by 100 and $95 \%$ ethanol ( 3 min each). After washing in $2 X$ SSC, sections were incubated overnight at $37^{\circ} \mathrm{C}$ in a humidified chamber with $12.5 \mu \mathrm{M}$ probe and RT97 anti-NF (1:100) in hybridization buffer. The following day, sections were washed in 2X SSC + $10 \%$ formamide at $37^{\circ} \mathrm{C}$ for $30 \mathrm{~min}$, followed by two incubations in $2 \mathrm{XSSC}$ for $5 \mathrm{~min}$ each. Sections were then briefly rinsed in PBS $+1 \%$ Triton-X100, and then incubated for in donkey anti-mouse FITC antibody diluted in $10 \mathrm{X}$ blocking buffer (1:100; Roche) + $0.3 \%$ Triton-X100 for $1 \mathrm{~h}$. Sections were finally washed in PBS for 5 min, post-fixed in 2 \% PFA for 15 min, washed 3 times in PBS (5 min each), rinsed in DEPCtreated water, and mounted under glass coverslips.

smFISH and IF signals were imaged using Leica SP8X as above. 63X/NA 1.4 objective and pulsed white light laser was used for imaging RNA in both culture and tissue samples. Scramble probe was used to set the image acquisition parameter that would not acquire any nonspecific signal from scramble probe. Taking XYZ image stacks at least two locations in each section scanned nerve sections.

Detection of nascently synthesized proteins - Rat sciatic nerve from three animals per condition was either left naïve or in vitro crushed and incubated in DMEM medium containing, $10 \%$ FBS + Cyclosporin A (20 $\mu \mathrm{M}$; Sigma $)+1 \%$ penicillin/streptomycin. Nerves were treated with $200 \mu \mathrm{g} / \mathrm{ml}$ anisomycin or vehicle (0.1\% DMSO) for $3 \mathrm{~h}$ at $37^{\circ} \mathrm{C}$, followed by adding $100 \mu \mathrm{g} / \mathrm{ml}$ O-propargyl-puromycin (OPP; 


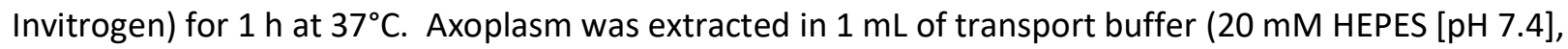
$100 \mathrm{mM}$ sodium acetate, $5 \mathrm{mM}$ magnesium acetate), after extraction SDS added to $1 \%$. Protein concentration was quantified by BCA assay and $350 \mu \mathrm{g}$ of total protein was used for biotin conjugation by click chemistry (100 $\mu \mathrm{M}$ biotin-PEG3-azide) according to manufacturer's instruction. The reaction mix was incubated for $2 \mathrm{~h}$ at room temperature on a rotator. 5 volumes ice-cold acetone was added to precipitate the protein. Protein pellets were resuspended in PBS containing $1 \%$ SDS. Streptavidin pulldown was carried out overnight at $4^{\circ} \mathrm{C}$ in $1 \mathrm{ml}$ volume containing $60 \mu \mathrm{l}$ of streptavidin magnetic beads (Life Technologies), $1 \%$ NP40, $0.1 \%$ SDS and 1x Complete EDTA-free protease inhibitor cocktail (Roche) in PBS. $10 \%$ of protein used for pull-down was taken to generate input samples. Beads were washed three times for 10 min with $1 \%$ NP40, $0.1 \%$ SDS in PBS at room temperature. Proteins were eluted from streptavidin beads by boiling for $10 \mathrm{~min}$ in $2 \mathrm{x}$ Laemmli sample buffer and then adjusted to $1 \mathrm{x}$ with PBS for denaturing polyacrylamide gel electrophoresis (SDS/PAGE).

Immunoblotting - Adult mouse DRG cultures (3 days in vitro, 80,000 neurons/well) were lysed in Laemmli sample buffer and denatured by boiling at $95^{\circ} \mathrm{C} \times 5 \mathrm{~min}$. Rat axoplasm from naïve and crushed sciatic nerves was extruded in nuclear transport buffer (20 mM HEPES [pH 7.3], 110 mM potassium acetate, $5 \mathrm{mM}$ magnesium acetate, supplemented with protease inhibitors) as previously described (Hanz et al., 2003). Lysates were cleared of debris by centrifugation at $15,000 \times \mathrm{g}$ for $15 \mathrm{~min}$ at $4^{\circ} \mathrm{C}$ and then normalized for protein content using Bradford assay (BioRad). Normalized protein lysates were fractionated by $10 \%$ SDS/PAGE and transferred onto a PVDF membrane (GE Healthcare Life Sciences, Marlborough, MA). After blocking in 5\% non-fat dried milk powder (Biorad) diluted in Tris-buffered saline with $1 \%$ Tween 20 (TBST), membranes were probed overnight at $4^{\circ} \mathrm{C}$ with Rabbit anti-KHSRP (1:1000; Novus) or Rabbit anti-GAPDH (1:2000; Cell Signaling Tech, Beverly, MA), Streptavidin-HRP (1:10000, Abcam) antibodies diluted in blocking buffer. Blots were washed in TBST and then incubated with horseradish peroxidase (HRP)-conjugated anti-rabbit IgG (1:2000; Cell Signaling Tech) diluted in 
blocking buffer for $1 \mathrm{~h}$ at room temperature. Blots were washed in TBST and signals were detected by $\mathrm{ECL}_{\text {Prime }}^{\mathrm{TM}}$ (GE Healthcare Life Sciences).

Fluorescence recovery after photobleaching - FRAP experiments were conducted at $37^{\circ} \mathrm{C}, 5 \% \mathrm{CO}_{2}$ as previously described (Yudin et al., 2008). Briefly, dissociated adult mouse DRG cultures transfected with the GFP ${ }^{M Y R} 5^{\prime} / 3^{\prime}$ khsrp were equilibrated in culture medium as above except phenol red was excluded. A region of interest (ROI) in the most distal axon of dissociated DRG neurons was photobleached with 488 $\mathrm{nm}$ argon laser set at $100 \%$ power for 80 frames at $0.65 \mathrm{sec}$ each. Pre-bleach and post-bleach signals were captured using $70 \%$ power for $488 \mathrm{~nm}$ laser line every $30 \mathrm{sec}$ ( 2 for pre-bleach and 30 for postbleach). Leica SP8X confocal microscope was used for imaging at $37^{\circ} \mathrm{C}, 5 \% \mathrm{CO}_{2}$ with $63 \mathrm{X} / \mathrm{NA} 1.4$ objective. Pinhole was set to 3 Airy units for pre-bleach, bleach, and post-bleach sequences to ensure full thickness excitation of the axon. ROls were $40 \times 40 \mu \mathrm{m}$ and at least $250 \mu \mathrm{m}$ from the soma.

Image analyses - Image J was used to quantify protein and RNA levels in sciatic nerve tissues from optical planes of XYZ scans. Axon only signal was extracted via Colocalization plug-in that extracted only protein or RNA signals that overlapped with axonal marker (NF) in each plane. Extracted 'axon only signal 'was projected as a separate channel (Kalinski et al., 2015). Signal intensities were then calculated from each XY plane of these axon only channels. NF immunoreactivity area was used to normalize signal intensities across the individual XY planes. The relative signal intensity was then averaged for all tiles in each biological replicate.

To assess regeneration in vivo, tile scans were post-processed by Straighten plug-in for ImageJ (http://imagej.nih.gov/ij/). SCG10 fluorescence intensity was measured along the length of the nerve using ImageJ. Regeneration index was calculated by measuring the average SCG10 intensity in bins across at least $3 \mathrm{~mm}$ distal to the crush site. The crush site was defined by the position along the nerve length with maximal SCG10 intensity (secondarily confirmed by DAPI signals and DIC images). 
For analyses of axon growth in vitro, dissociated DRGs were immunostained with NF antibodies as described above. Images from 36 or $48 \mathrm{~h}$ cultures were used for neurite length and branching parameters (neurites/cell body and branch density) using WIS-Neuromath (Rishal and Fainzilber, 2014).

For NMJs, confocal Z stacks from muscle were projected as single XY images using ImageJ. Nerve terminal and endplate (AchRs) areas were calculated by Image $J$ and fractional occupancy of NMJ was calculated by dividing nerve terminal area to endplate AChR area as described (Maimon et al., 2018).

For FRAP image analyses, raw images sequences were analyzed for recovery in the bleached ROI using Leica confocal software package. Recovery was determined relative to pre-bleach and post-bleach signals, which were set at 100 and $0 \%$ to allow for comparisons between experiments and between neurons.

Statistical analyses - GraphPad Prism software package (La Jolla, CA) was used for statistical analyses. One-way ANOVA with Tukey post-hoc was used to compare between data points and Student's t-test was used to compare two independent groups for most experiments. For FRAP studies, two-way ANOVA with Tukey post-hoc was used, where control values were compared to cycloheximide- and anisomycin-treated cultures for each time point. All experiments were performed in at least triplicate. $\mathrm{P} \leq 0.05$ was considered as statistically significant. 


\section{REFERENCES}

Aakalu, G., Smith, W.B., Nguyen, N., Jiang, C., and Schuman, E.M. (2001). Dynamic visualization of local protein synthesis in hippocampal neurons. Neuron 30, 489-502.

Aschrafi, A., Kar, A.N., Natera-Naranjo, O., MacGibeny, M.A., Gioio, A.E., and Kaplan, B.B. (2012). MicroRNA-338 regulates the axonal expression of multiple nuclear-encoded mitochondrial mRNAs encoding subunits of the oxidative phosphorylation machinery. Cell Mol Life Sci 69, 4017-4027.

Barrientos, S.A., Martinez, N.W., Yoo, S., Jara, J.S., Zamorano, S., Hetz, C., Twiss, J.L., Alvarez, J., and Court, F.A. (2011). Axonal degeneration is mediated by the mitochondrial permeability transition pore. J Neurosci 31, 966-978.

Batista, A.F.R., Martinez, J.C., and Hengst, U. (2017). Intra-axonal Synthesis of SNAP25 Is Required for the Formation of Presynaptic Terminals. Cell Rep 20, 3085-3098.

Beckel-Mitchener, A.C., Miera, A., Keller, R., and Perrone-Bizzozero, N.I. (2002). Poly(A) tail lengthdependent stabilization of GAP-43 mRNA by the RNA-binding protein HuD. J Biol Chem 277, 2799628002.

Ben-Yaakov, K., Dagan, S.Y., Segal-Ruder, Y., Shalem, O., Vuppalanchi, D., Willis, D.E., Yudin, D., Rishal, I., Rother, F., Bader, M., et al. (2012). Axonal transcription factors signal retrogradely in lesioned peripheral nerve. EMBO J 31, 1350-1363.

Bird, C.W., Gardiner, A.S., Bolognani, F., Tanner, D.C., Chen, C.Y., Lin, W.J., Yoo, S., Twiss, J.L., and Perrone-Bizzozero, N. (2013). KSRP modulation of GAP-43 mRNA stability restricts axonal outgrowth in embryonic hippocampal neurons. PLoS One 8, e79255.

Bolognani, F., Merhege, M.A., Twiss, J., and Perrone-Bizzozero, N.I. (2004). Dendritic localization of the RNA-binding protein HuD in hippocampal neurons: association with polysomes and upregulation during contextual learning. Neurosci Lett 371, 152-157.

Cavalli, V., Kujala, P., Klumperman, J., and Goldstein, L.S. (2005). Sunday Driver links axonal transport to damage signaling. J Cell Biol 168, 775-787.

Cho, Y., Sloutsky, R., Naegle, K.M., and Cavalli, V. (2013). Injury-induced HDAC5 nuclear export is essential for axon regeneration. Cell 155, 894-908.

Colak, D., Ji, S.J., Porse, B.T., and Jaffrey, S.R. (2013). Regulation of axon guidance by compartmentalized nonsense-mediated mRNA decay. Cell 153, 1252-1265.

Dalla Costa, I., Buchanan, C., Zdradzinski, M.D., Sahoo, P.K., Smith, T.P., Thames, E., Kar, A.N., and Twiss, J.L. (2020). Functional platforms for organizing axonal mRNA transport and translation. Nat Rev Neurosci in press.

Donnelly, C.J., Park, M., Spillane, M., Yoo, S., Pacheco, A., Gomes, C., Vuppalanchi, D., McDonald, M., Kim, H.H., Merianda, T.T., et al. (2013). Axonally synthesized beta-actin and GAP-43 proteins support distinct modes of axonal growth. J Neurosci 33, 3311-3322.

Donnelly, C.J., Willis, D.E., Xu, M., Tep, C., Jiang, C., Yoo, S., Schanen, N.C., Kirn-Safran, C.B., van Minnen, J., English, A., et al. (2011). Limited availability of ZBP1 restricts axonal mRNA localization and nerve regeneration capacity. EMBO J 30, 4665-4677. 
Forester, C.M., Zhao, Q., Phillips, N.J., Urisman, A., Chalkley, R.J., Oses-Prieto, J.A., Zhang, L., Ruggero, D., and Burlingame, A.L. (2018). Revealing nascent proteomics in signaling pathways and cell differentiation. Proc Natl Acad Sci U S A 115, 2353-2358.

Gherzi, R., Lee, K.Y., Briata, P., Wegmuller, D., Moroni, C., Karin, M., and Chen, C.Y. (2004). A KH domain RNA binding protein, KSRP, promotes ARE-directed mRNA turnover by recruiting the degradation machinery. Mol Cell 14, 571-583.

Gomes, C., Lee, S.J., Gardiner, A.S., Smith, T., Sahoo, P.K., Patel, P., Thames, E., Rodriguez, R., Taylor, R., Yoo, S., et al. (2017). Axonal localization of neuritin/CPG15 mRNA is limited by competition for HuD binding. J Cell Sci 130, 3650-3662.

Hanz, S., Perlson, E., Willis, D., Zheng, J.Q., Massarwa, R., Huerta, J.J., Koltzenburg, M., Kohler, M., vanMinnen, J., Twiss, J.L., et al. (2003). Axoplasmic importins enable retrograde injury signaling in lesioned nerve. Neuron 40, 1095-1104.

Hengst, U., Cox, L.J., Macosko, E.Z., and Jaffrey, S.R. (2006). Functional and selective RNA interference in developing axons and growth cones. J Neurosci 26, 5727-5732.

Kalinski, A.L., Sachdeva, R., Gomes, C., Lee, S.J., Shah, Z., Houle, J.D., and Twiss, J.L. (2015). mRNAs and Protein Synthetic Machinery Localize into Regenerating Spinal Cord Axons When They Are Provided a Substrate That Supports Growth. J Neurosci 35, 10357-10370.

Kar, A., Lee, S., and Twiss, J. (2018). Expanding Axonal Transcriptome Brings New Functions for Axonally Synthesized Proteins in Health and Disease. The Neuroscientist 24, 111-129.

Kim, H.H., Kim, P., Phay, M., and Yoo, S. (2015). Identification of precursor microRNAs within distal axons of sensory neuron. J Neurochem 134, 193-199.

Lee, S., Sahoo, P., Oses-Prieto, J., Kawaguchi, R., Kar, A., Oliver, D., Chand, S., Shtutman, M., Rozenbaum, M., Miller-Randolph, S., et al. (2018). hnRNPs binding to the axonal localization motifs of Nrn1 and HMGB1 mRNAs define growth-associated RNA regulons. Mol Cell Proteomics 17, 2091-2106.

Li, X., Lin, W.J., Chen, C.Y., Si, Y., Zhang, X., Lu, L., Suswam, E., Zheng, L., and King, P.H. (2012). KSRP: a checkpoint for inflammatory cytokine production in astrocytes. Glia 60, 1773-1784.

Lin, W.J., Zheng, X., Lin, C.C., Tsao, J., Zhu, X., Cody, J.J., Coleman, J.M., Gherzi, R., Luo, M., Townes, T.M., et al. (2011). Posttranscriptional control of type I interferon genes by KSRP in the innate immune response against viral infection. Mol Cell Biol 31, 3196-3207.

MacLean, B., Tomazela, D.M., Shulman, N., Chambers, M., Finney, G.L., Frewen, B., Kern, R., Tabb, D.L., Liebler, D.C., and MacCoss, M.J. (2010). Skyline: an open source document editor for creating and analyzing targeted proteomics experiments. Bioinformatics 26, 966-968.

Maimon, R., Ionescu, A., Bonnie, A., Sweetat, S., Wald-Altman, S., Inbar, S., Gradus, T., Trotti, D., Weil, M., Behar, O., et al. (2018). miR126-5p Downregulation Facilitates Axon Degeneration and NMJ Disruption via a Non-Cell-Autonomous Mechanism in ALS. J Neurosci 38, 5478-5494.

Merianda, T.T., Coleman, J., Kim, H.H., Kumar Sahoo, P., Gomes, C., Brito-Vargas, P., Rauvala, H., Blesch, A., Yoo, S., and Twiss, J.L. (2015). Axonal amphoterin mRNA is regulated by translational control and enhances axon outgrowth. J Neurosci 35, 5693-5706.

Merianda, T.T., Gomes, C., Yoo, S., Vuppalanchi, D., and Twiss, J.L. (2013). Axonal localization of neuritin/CPG15 mRNA in neuronal populations through distinct 5' and 3' UTR elements. J Neurosci 33, 13735-13742. 
Min, H., Turck, C.W., Nikolic, J.M., and Black, D.L. (1997). A new regulatory protein, KSRP, mediates exon inclusion through an intronic splicing enhancer. Genes \& Devel 11, 1023-1036.

Murashov, A.K., Chintalgattu, V., Islamov, R.R., Lever, T.E., Pak, E.S., Sierpinski, P.L., Katwa, L.C., and Van Scott, M.R. (2007). RNAi pathway is functional in peripheral nerve axons. FASEB J 21, 656-670.

Olguin, S.L., Patel, P., Dell'Orco, M., Gardiner, A.S., Cole, R., Buchanan, C., Sundara, A., Mudge, J., Allan, A.M., Ortinski, P., et al. (2020). The RNA binding protein KHSRP attenuates axonal and dendritic growth, synaptic transmission, and memory consolidation via dysregulation of neuronal gene expression. BioRxiv doi.org/10.1101/2020.10.25.354076.

Osen-Sand, A., Catsicas, M., Staple, J.K., Jones, K.A., Ayala, G., Knowles, J., Grenningloh, G., and Catsicas, S. (1993). Inhibition of axonal growth by SNAP-25 antisense oligonucleotides in vitro and in vivo. Nature 364, 445-448.

Pacheco, A., Merianda, T.T., Twiss, J.L., and Gallo, G. (2020). Mechanism and role of the intra-axonal Calreticulin translation in response to axonal injury. Exp Neurol 323, 113072.

Pan, F., Huttelmaier, S., Singer, R.H., and Gu, W. (2007). ZBP2 facilitates binding of ZBP1 to beta-actin mRNA during transcription. Mol Cell Biol 27, 8340-8351.

Pascale, A., Gusev, P.A., Amadio, M., Dottorini, T., Govoni, S., Alkon, D.L., and Quattrone, A. (2004). Increase of the RNA-binding protein HuD and posttranscriptional up-regulation of the GAP-43 gene during spatial memory. Proc Natl Acad Sci U S A 101, 1217-1222.

Perlson, E., Hanz, S., Ben-Yaakov, K., Segal-Ruder, Y., Seger, R., and Fainzilber, M. (2005). Vimentindependent spatial translocation of an activated MAP kinase in injured nerve. Neuron 45, 715-726.

Perry, R.B., Rishal, I., Doron-Mandel, E., Kalinski, A.L., Medzihradszky, K.F., Terenzio, M., Alber, S., Koley, S., Lin, A., Rozenbaum, M., et al. (2016). Nucleolin-Mediated RNA Localization Regulates Neuron Growth and Cycling Cell Size. Cell Rep 16, 1664-1676.

Rishal, I., and Fainzilber, M. (2014). Axon-soma communication in neuronal injury. Nat Rev Neurosci 15, 32-42.

Rishal, I., Michaelevski, I., Rozenbaum, M., Shinder, V., Medzihradszky, K.F., Burlingame, A.L., and Fainzilber, M. (2010). Axoplasm isolation from peripheral nerve. Dev Neurobiol 70, 126-133.

Sahoo, P., Kar, A., Samra, N., Terenzio, M., Patell, P., Lee, S., Miller, S., Thames, E., Jones, B., Kawaguchi, R., et al. (2020). A translational switch drives axonal stress granule disassembly through Casein Kinase 2a. Curr Biol in press (doi.org/10.1016/j.cub.2020.09.043).

Sahoo, P.K., Lee, S.J., Jaiswal, P.B., Alber, S., Kar, A.N., Miller-Randolph, S., Thames, E., Taylor, E.E., Smith, T., Singh, B., et al. (2018). Axonal G3BP1 stress granule protein limits axonal mRNA translation and nerve regeneration. Nat Commun 9, 3358.

Sharma, V., Eckels, J., Schilling, B., Ludwig, C., Jaffe, J.D., MacCoss, M.J., and MacLean, B. (2018). Panorama Public: A Public Repository for Quantitative Data Sets Processed in Skyline. Mol Cell Proteomics 17, 1239-1244.

Shin, J.E., Miller, B.R., Babetto, E., Cho, Y., Sasaki, Y., Qayum, S., Russler, E.V., Cavalli, V., Milbrandt, J., and DiAntonio, A. (2012). SCG10 is a JNK target in the axonal degeneration pathway. Proc Natl Acad Sci U S A 109, E3696-3705.

Skene, J.H. (1989). Axonal growth-associated proteins. Annu Rev Neurosci 12, 127-156. 
Smith, C.L., Afroz, R., Bassell, G.J., Furneaux, H.M., Perrone-Bizzozero, N.I., and Burry, R.W. (2004). GAP43 mRNA in growth cones is associated with HuD and ribosomes. J Neurobiol 61, 222-235.

Smith, D.S., and Skene, P. (1997). A transcription-dependent switch controls competence of adult neurons for distinct modes of axon growth. J Neurosci 17, 646-658.

Smith, T.P., Sahoo, P.K., Kar, A.N., and Twiss, J.L. (2020). Intra-axonal mechanisms driving axon regeneration. Brain Res 1740, 146864.

Sollner, T., Bennett, M.K., Whiteheart, S.W., Scheller, R.H., and Rothman, J.E. (1993). A protein assembly-disassembly pathway in vitro that may correspond to sequential steps of synaptic vesicle docking, activation, and fusion. Cell 75, 409-418.

Terenzio, M., Koley, S., Samra, N., Rishal, I., Zhao, Q., Sahoo, P.K., Urisman, A., Marvaldi, L., Oses-Prieto, J.A., Forester, C., et al. (2018). Locally translated mTOR controls axonal local translation in nerve injury. Science 359, 1416-1421.

Trabucchi, M., Briata, P., Garcia-Mayoral, M., Haase, A.D., Filipowicz, W., Ramos, A., Gherzi, R., and Rosenfeld, M.G. (2009). The RNA-binding protein KSRP promotes the biogenesis of a subset of microRNAs. Nature 459, 1010-1014.

Twiss, J., Smith, D., Chang, B., and Shooter, E. (2000a). Translational control of ribosomal protein L4 is required for rapid neurite extension. Neurobiol Dis 7, 416-428.

Twiss, J.L., Smith, D.S., Chang, B., and Shooter, E.M. (2000b). Translational control of ribosomal protein L4 mRNA is required for rapid neurite regeneration. Neurobiol Dis 7, 416-428.

Urisman, A., Levin, R.S., Gordan, J.D., Webber, J.T., Hernandez, H., Ishihama, Y., Shokat, K.M., and Burlingame, A.L. (2017). An Optimized Chromatographic Strategy for Multiplexing In Parallel Reaction Monitoring Mass Spectrometry: Insights from Quantitation of Activated Kinases. Mol Cell Proteomics 16, 265-277.

Van der Zee, C.E., Nielander, H.B., Vos, J.P., Lopes da Silva, S., Verhaagen, J., Oestreicher, A.B., Schrama, L.H., Schotman, P., and Gispen, W.H. (1989). Expression of growth-associated protein B-50 (GAP43) in dorsal root ganglia and sciatic nerve during regenerative sprouting. J Neurosci 9, 3505-3512.

Vargas, J.N., Kar, A.N., Kowalak, J.A., Gale, J.R., Aschrafi, A., Chen, C.Y., Gioio, A.E., and Kaplan, B.B. (2016). Axonal localization and mitochondrial association of precursor microRNA 338. Cell Mol Life Sci 73, 4327-4340.

Willis, D.E., and Twiss, J.L. (2011). Profiling axonal mRNA transport. Methods Mol Biol 714, 335-352.

Willis, D.E., van Niekerk, E.A., Sasaki, Y., Mesngon, M., Merianda, T.T., Williams, G.G., Kendall, M., Smith, D.S., Bassell, G.J., and Twiss, J.L. (2007). Extracellular stimuli specifically regulate localized levels of individual neuronal mRNAs. J Cell Biol 178, 965-980.

Yoo, S., Kim, H.H., Kim, P., Donnelly, C.J., Kalinski, A.L., Vuppalanchi, D., Park, M., Lee, S.J., Merianda, T.T., Perrone-Bizzozero, N.I., et al. (2013). A HuD-ZBP1 ribonucleoprotein complex localizes GAP-43 mRNA into axons through its 3' untranslated region AU-rich regulatory element. J Neurochem 126, 792804.

Yudin, D., Hanz, S., Yoo, S., lavnilovitch, E., Willis, D., Gradus, T., Vuppalanchi, D., Segal-Ruder, Y., BenYaakov, K., Hieda, M., et al. (2008). Localized regulation of axonal RanGTPase controls retrograde injury signaling in peripheral nerve. Neuron 59, 241-252. 
bioRxiv preprint doi: https://doi.org/10.1101/2020.10.26.356162; this version posted October 26, 2020. The copyright holder for this preprint

(which was not certified by peer review) is the author/funder, who has granted bioRxiv a license to display the preprint in perpetuity. It is made available under aCC-BY-NC-ND 4.0 International license.

Zhang, H.L., Eom, T., Oleynikov, Y., Shenoy, S.M., Liebelt, D.A., Dictenberg, J.B., Singer, R.H., and Bassell, G.J. (2001). Neurotrophin-induced transport of a beta-actin mRNP complex increases beta-actin levels and stimulates growth cone motility. Neuron 31, 261-275.

Zheng, J.Q., Kelly, T.K., Chang, B., Ryazantsev, S., Rajasekaran, A.K., Martin, K.C., and Twiss, J.L. (2001). A functional role for intra-axonal protein synthesis during axonal regeneration from adult sensory neurons. J Neurosci 21, 9291-9303. 


\section{FIGURE LEGENDS}

\section{Figure 1: Peripheral nerve injury changes the axonal RNA binding protein populations}

A-B) Sciatic nerve axoplasm harvested proximal to the injury site from 3-28 d post-crush lesion was digested and processed for liquid chromatography and mass spectrometry using parallel reaction monitoring (PRM) to detect proteins with known RNA binding activity. Levels of proteins from spectral counts relative to uninjured (naïve) axoplasm as shown as $\log _{2}$ fold-change as indicated in $\mathbf{A}$ ( $N=3$ for each time point). B shows volcano plot for PRM results for $7 \mathrm{~d}$ crush vs. naïve samples graphed as $\log _{2}$ fold-change vs. - $\log _{10} p$ value for differences. A subset of proteins are identified here.

C) Representative immunoblot for naïve and $7 \mathrm{~d}$ injured sciatic nerve axoplasm confirms the increase in FXR1, hnRNP A3, hnRNP AB, hnRNP H1, and KHSRP. ERK 1/2 and GAPDH show relatively equivalent loading of the lysates. Also see Suppl. Figure S1 for graphical representation of full time course.

D-E) Representative immunoblots for kinetics of KHSRP elevation in sciatic nerve axoplasm over 0-28 d post-crush lesion are shown in D. Quantification of KHSRP immunoreactivity across multiple animals is shown as mean fold-change relative to naïve \pm standard error of the mean (SEM; $N=3$ for each time point; ${ }^{* *}=\mathrm{P} \leq 0.001$ for vs. $0 \mathrm{~d}, \dagger=\mathrm{p} \leq 0.05,++\dagger=\mathrm{p} \leq 0.005,++\dagger=\mathrm{p} \leq 0.001$ and $+++\dagger=\mathrm{p} \leq 0.0005$ vs. $3 d, \& \& \&=p \leq 0.001$ and $\& \& \& \&=p \leq 0.0005$ vs. $7 d$, and $\$ \$=p \leq 0.01$ and $\$ \$ \$=p \leq 0.0005$ vs. $14 d$ by one way ANOVA with Tukey's post-hoc analysis).

F) Representative confocal images for KHSRP protein in naïve and $7 \mathrm{~d}$ post-crush sciatic nerve. Upper panels of each pair show XYZ maximum projection of merged KHSRP (grey) and neurofilament (NF; magenta) signals; lower panels show KHSRP signals overlapping with NF ('axonal KHSRP') in each individual Z plane projected as an XYZ image [Scale bar $=5 \mu \mathrm{m}$ ]. 


\section{Figure 2: KHSRP depletion enhances axonal growth from naïve but not injury-conditioned DRG}

neurons.

A) Representative images of $24 \mathrm{~h}$ DRG neuron cultures from naïve and $7 \mathrm{~d}$ injury-conditioned $K h s r p^{+/+}$ and $\mathrm{Khsp}^{-/}$mice immunostained for NF are shown [Scale bar $=100 \mu \mathrm{m}$ ].

B) Quantification of total axon length per neuron from cultures as in A shows significantly greater axon growth in $\mathrm{Khsr}^{-/}$compared to $\mathrm{Khsr}^{+/+}$DRGs. However, there is no significant difference in axon length between the genotypes when cultures were prepared $7 \mathrm{~d}$ after nerve crush injury (i.e., 'injuryconditioned 'neurons). Data are expressed as mean \pm SEM; refer to Supplemental Figure S2 for axon branching analyses ( $\geq 75$ neurons analyzed per sample over 3 independent cultures; ${ }^{*}=\mathrm{P} \leq 0.05$ and $* *$ $=\mathrm{P} \leq 0.01$ by one way ANOVA with Tukey's post-hoc analysis).

C) Representative immunoblot shows signal for KHSRP in cell body and axonal preparations from DRG cultures from indicated mice. There is no KHSRP band in the $K h s r p^{-/}$cell body or axonal preparations, despite GAPDH showing more protein in those samples than in the $K h s r p^{+/+}$DRGs.

\section{Figure 3: KHSRP deletion increases in vivo axon regeneration after a conditioning Sciatic nerve injury.}

A) Representative SCG10 immunostained images of sciatic nerves after single (3 d; upper panels) or injury-conditioned (7+3d; lower panels) sciatic nerve crush injuries for $K h s r p^{+/+}$and $K h s r p^{-/}$are shown. Proximal is on the left and distal on the right; the dashed line indicates the injury site, with the second injury for the double crush injured animals placed at $\sim 0.5 \mathrm{~cm}$ proximal to the initial injury site [Scale bar $=500 \mu \mathrm{m}]$.

B) Regeneration indices calculated as fraction of SCG10 axonal profiles at injury site $(0 \mu \mathrm{m})$ are shown as mean \pm SEM as indicated. There was no significant difference in the regeneration after the single injury, but the injury-conditioned $\mathrm{Khsr}^{-{ }^{-}}$mice show significantly higher regeneration indices ( $\mathrm{N}=5$ mice per genotype and condition; ${ }^{*}=\mathrm{P} \leq 0.05, * *=\mathrm{P} \leq 0.01$, and $* * *=\mathrm{P} \leq 0.001$ by TWO way ANOVA with Bonferroni post-hoc analysis). 
C) Confocal XYZ images of gastrocnemius muscles of the injury-conditioned $K h s r p^{+/+}$and $K h s r p^{-/}$mice at

$14 \mathrm{~d}$ after second nerve crush are shown. NMJs are detected by post-synaptic ( $\alpha$-bungarotoxin; green) and pre-synaptic markers (cocktail of anti-NF, -synapsin I, and -synaptophysin; red) signals showing higher matching of pre- and post-synaptic markers in $K h s r p^{-/-}$than $K h s r p^{+/+}$mice [Scale bar $=20 \mu \mathrm{m}$ ].

D) Quantification of NMJ occupancy (\% presynaptic area/postsynaptic area) shows significantly greater occupancy in the injury-conditioned $\mathrm{Khsp}^{-/-}$mice but no difference with the single crush lesion (injuryconditioned $=7+14 \mathrm{~d}$; single nerve crush $=14 \mathrm{~d})$. Data are expressed as mean \pm SEM $(\mathrm{N} \geq 15$ NMJs quantified in 3 animals per condition per genotype; ${ }^{* *}=\mathrm{P} \leq 0.01$ by Student's $\mathrm{t}$ test).

E) Schematic for the culture system to allow for axotomy and regeneration from the injured axon rather than initiation of axon growth from the cell body as seen in cultures of in vivo injury-conditioned neurons. For this, dissociated DRGs are grown on a porous membrane for $36 \mathrm{~h}$. Axons are then severed by scraping the undersurface of the membrane. Axon regrowth along the undersurface of the membrane was assessed $72 \mathrm{~h}$ later.

F) Representative Neurolucida tracings for NF-stained axons from filter undersurface for Khsrp ${ }^{+/}$and Khsrp ${ }^{--}$DRGs at $72 \mathrm{~h}$ post in vitro axotomy [Scale bar $=100 \mu \mathrm{m}$ ].

G) Quantification of summed axon lengths per filter from $\mathrm{G}$ are shown as mean $\pm \mathrm{SEM}$. The Khsrp $^{-/}$DRG cultures show significantly more in vitro axon regeneration than $K H S R P^{+/+}$DRG cultures $(\mathrm{N}=3$ for each genotype with $\geq 17$ random fields analyzed; ${ }^{* *}=\mathrm{P} \leq 0.001$ by Student's $t$-test).

\section{Figure 4: Khsrp mRNA is transported into PNS axons.}

A) Representative confocal images for smFISH/IF for Khsrp mRNA (grey) and NF (magenta) in dissociated DRG cultures are shown as indicated. There is a clear signal for Khsrp mRNA in the cell body (left and middle columns) and distal axons (right column) of DRGs from $K h s r p^{+/+}$mice. Khsrp ${ }^{-/}$DRGs showed no Khsrp mRNA in the axons; however, there was faint but consistent signal in the cell body [Scale bar $=10$ $\mu \mathrm{m}]$. 
B-C) Quantification smFISH signals for Khsrp mRNA in cell bodies (B) and axons (C) is shown as mean \pm SEM for scramble $\left(K_{h s r p^{+/+}}\right)$and $K h s r p$ mRNA $\left(K_{s r p^{+/+}}\right.$and $\left.K h s r p^{-/-}\right)$probes; in each case, scramble probe was hybridized to $\mathrm{Khsr}^{+/+}$DRG cultures as in panel $\mathrm{A}\left(\mathrm{N} \geq 16\right.$ neurons over three separate cultures; ${ }^{*}=\mathrm{P}$ $\leq 0.05, * *=\mathrm{P} \leq 0.01$, and $* * *=\mathrm{P} \leq 0.001$ by one way ANOVA with Tukey's post-hoc analysis).

D) Representative confocal images for smFISH/IF for Khsrp mRNA (grey) and NF (magenta) in uninjured sciatic nerve are shown as indicated. Left column shows XYZ projections from eight optical planes taken at $0.2 \mu \mathrm{m} \mathrm{Z} \mathrm{step} \mathrm{interval;} \mathrm{right} \mathrm{column} \mathrm{shows} \mathrm{'axon} \mathrm{only} \mathrm{'Khsrp} \mathrm{mRNA} \mathrm{signals} \mathrm{generated} \mathrm{by} \mathrm{extracting}$ FISH signals overlapping with NF in individual Z sections and projecting those as an 'Axonal Khsrp mRNA' $\mathrm{XYZ}$ image [Scale bar $=5 \mu \mathrm{m}]$.

E) Quantification of axonal Khsrp mRNA signals from $D$ are shown as mean \pm SEM ( $N=6$ animals per genotype; $^{* *}=\mathrm{P} \leq 0.01$ by Student's $t$-test).

\section{Figure 5: Khsrp mRNA is rapidly translated in axons after injury}

A) Schematic of nerve ligation model used for panels B-C. Proximal nerve is on the left and distal on the right as indicated. The nerve was ligated and then immediately crushed $\sim 1 \mathrm{~cm}$ distal to the ligation. B) Confocal images for KHSRP protein in naïve and post-crush injury ( 3 and $16 \mathrm{~h}$ ). Left column shows XYZ projections of merged signals for KHSRP (grey) and NF (magenta); right column shows 'axonal KHSRP' signals as from individual Z planes that were projected as an XYZ image. Representative images for ligation efficiency and KHSRP signals proximal and distal to ligation are shown in Suppl. Figure S4 [Scale bar $=5 \mu \mathrm{m}]$.

C) Quantification of the axonal KHSRP immunoreactivity from ligation proximal and distal and crush sites are shown as mean \pm SEM $\left(N \geq 3\right.$ animals per time point; $*=p \leq 0.05$ and ${ }^{* *}=p \leq 0.01$ for indicated time points vs. naïve nerve, $+\dagger=p \leq 0.01$ for indicated sample vs. crush proximal, and $\& \&=p \leq 0.01$ for indicated sample vs. crush distal by Student's $t$-test; ligation proximal vs. distal have no significance). 
D) Representative immunoblots for ex vivo puromycinylated naïve vs. crushed sciatic nerve segments are shown as indicated. Protein synthesis inhibition with anisomycin completely blocks the puromycinylation of KHSRP in axoplasm samples extruded from the nerve segments, and GAPDH shows relatively equivalent protein loading across the lanes.

E) Quantification of puromycinylated KHSRP signals from D is shown as mean \pm SEM. Crush injury significantly increases axonal KHSRP synthesis and this blocked by anisomycin $\left(\mathrm{N}=3{ }^{* * *}=\mathrm{P} \leq 0.001\right.$ by one way ANOVA with Tukey's post-hoc analysis).

F) FRAP analyses for distal axons of neurons transfected with GFP ${ }^{M Y R} 5^{\prime} / 3^{\prime}$ khsrp (see schematic at top) is shown as normalized average $\%$ recovery \pm SEM. Pre-treatment treated with anisomycin or cycloheximide significantly attenuates the GFP recovery, indicating protein synthesis dependent recovery in the axons $\left(\mathrm{N} \geq 30\right.$ neurons over 3 repetitions, $* p \leq 0.05, * * p \leq 0.01$, and ${ }^{* * *} p \leq 0.005$ by two-way ANOVA with Bonfieri post-hoc analyses for indicated time points vs. control). Representative images sequences for FRAP are shown in Suppl. Figure S5.

Figure 6: The ARE- binding KH4 domain of KHSRP attenuates axon growth and axonal mRNA levels.

A-C) Sciatic nerve levels of Gap43 (A) and Snap25 (B) mRNA levels are increased $7 \mathrm{~d}$ after sciatic nerve crush in $\mathrm{Khsrp}^{-/}$compared to $\mathrm{Khsrp}^{+/+}$mice. Sciatic nerve Hmgb1 mRNA levels (C) show no change comparing the $\mathrm{Khsrp}^{-/-}$vs. $\mathrm{Khsr}^{+/+}$mice or with injury. All genotypes show a significant increase for Gap43 mRNA with crush injury. Values shown as mean \pm SEM $\left(\mathrm{N}=5\right.$ mice per genotype; ${ }^{*}=\mathrm{P} \leq 0.05$ for indicated conditions by two-way ANOVA with Bonferroni post-hoc; $\&=P \leq 0.05, \& \&=P \leq 0.01$, and $\& \& \&=\mathrm{P} \leq 0.005$ and NS = not significant for crush vs. naïve within genotype by Student's t-test).

D) Representative images of DRG neurons transfected with GFP, GFP-KHSRP, or GFP-KHSRP $\triangle$ KH4 are shown for $\mathrm{KhSrp}^{+/+}$and $\mathrm{Khsrp}^{-/-}$(schematics for constructs above image columns) as indicated [Scale bar $=100 \mu \mathrm{m}]$. 
E) Quantification of total axon length/neuron for DRG cultures transfected as in D are shown as mean \pm

SEM. Expression of GFP-KHSRP decreases axon outgrowth in both $K h s r p^{+/+}$and $K h s r p^{-/}$DRGs, but GFP-

KHSRP $\triangle$ KH4 had no significant effect on $\mathrm{Khsr}^{+/+}$and only a modest decrease in axon length in $\mathrm{Khsr}^{-/}$

neurons $\left(\mathrm{N} \geq 30\right.$ neurons over three different culture preparations; ${ }^{*}=\mathrm{P} \leq 0.05,{ }^{* *}=\mathrm{P} \leq 0.01$, and ${ }^{* * *}=$ $\mathrm{P} \leq 0.001$ by one-way ANOVA with Tukey's post-hoc analysis for indicated comparisons).

F-G) Analysis of axonal (F) and cell body (G) Gap43 mRNA levels in neurons transfected as in D-E is shown as mean mRNA copies/ng of total RNA \pm SEM after normalization to mitochondrial 12S RNA $(\mathrm{N}=$ 3 per genotype and per condition; ${ }^{*}=\mathrm{P} \leq 0.05$ and ${ }^{* *}=\mathrm{P} \leq 0.001$ by one-way ANOVA with Tukey's post-hoc analysis for indicated comparisons). 
Figure 1
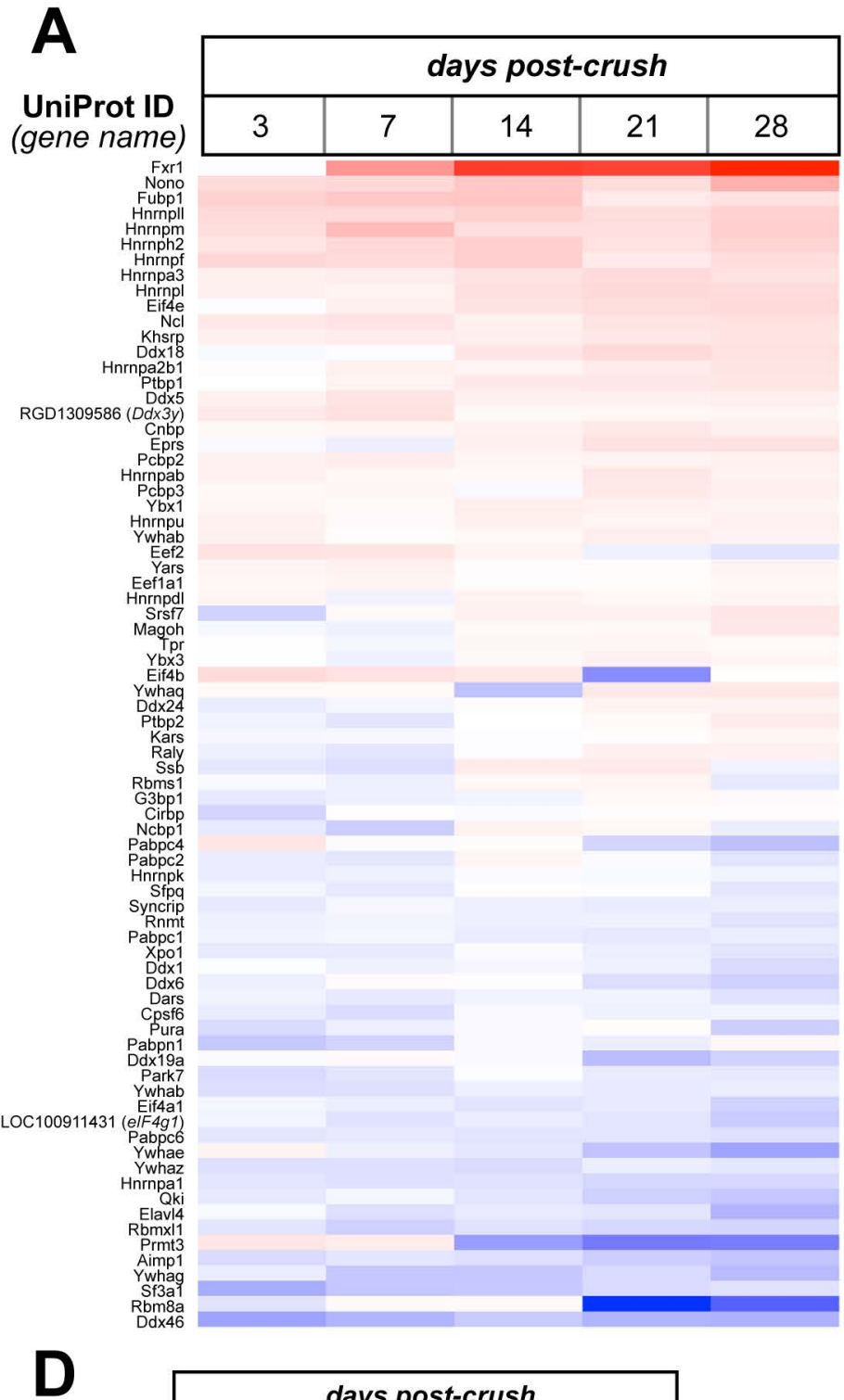

B

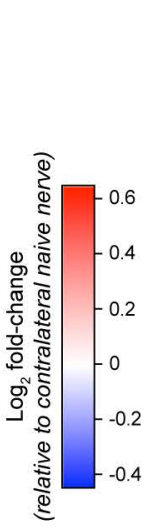

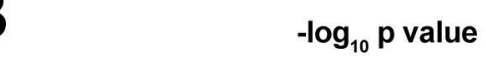

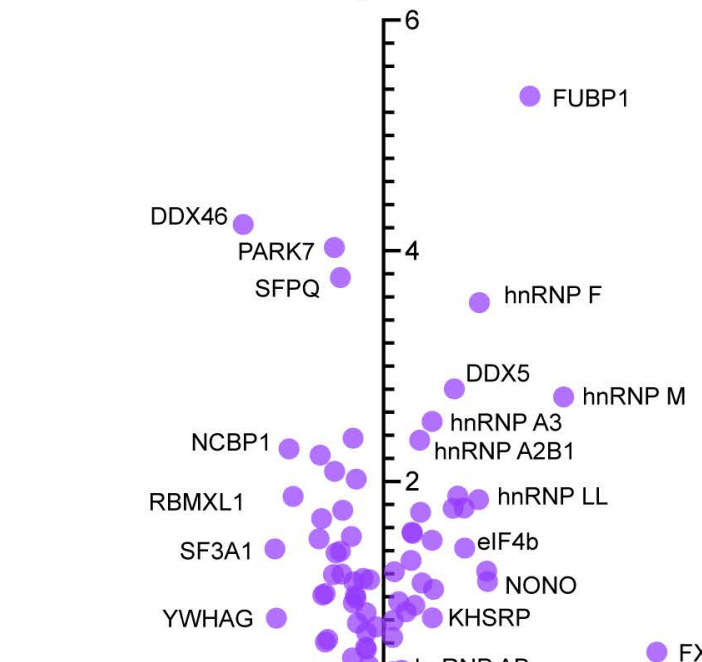

PABPN1

FXR1

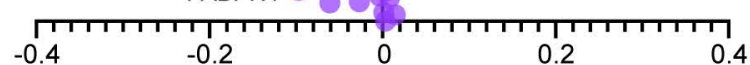

$\log _{2}$ Fold Change
$(7 d$ crush vs. naive)

C

days post-

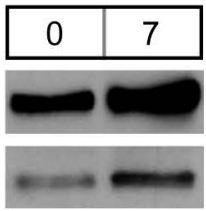

FXR1

crush

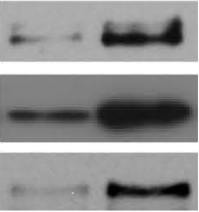

hnRNPA3

$h n R N P A B$

hnRNP H1

KHSRP

KHSRP (80 kDa)

GAPDH (37 kDa)

\begin{tabular}{|l|l|l|l|l|l|}
\hline \multicolumn{6}{|c|}{ days post-crush } \\
\hline 0 & 3 & 7 & 14 & 21 & 28 \\
\hline
\end{tabular}

ERK1/2

GAPDH

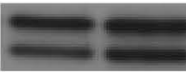

E

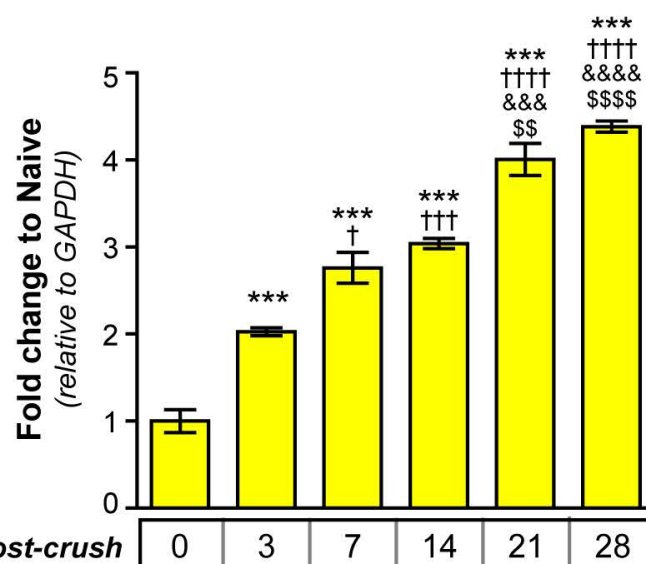

\begin{tabular}{|l|l|l|l|l|l|}
0 & 3 & 7 & 14 & 21 & 28 \\
\hline
\end{tabular}

\section{F Naive}
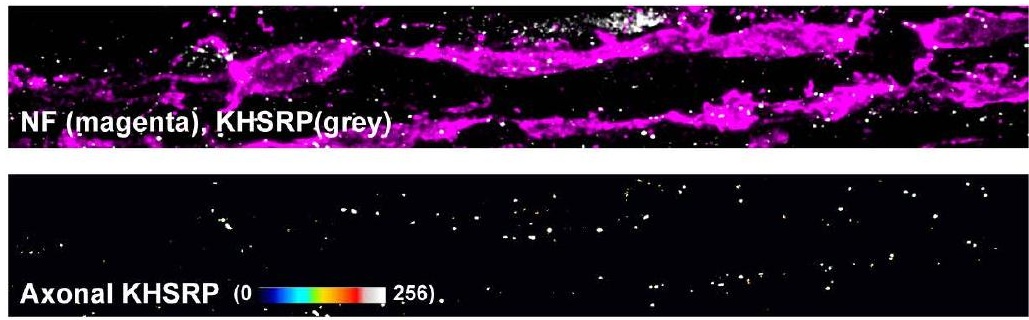

7 days post-crush

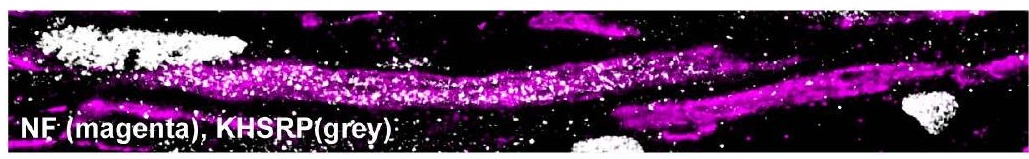

W

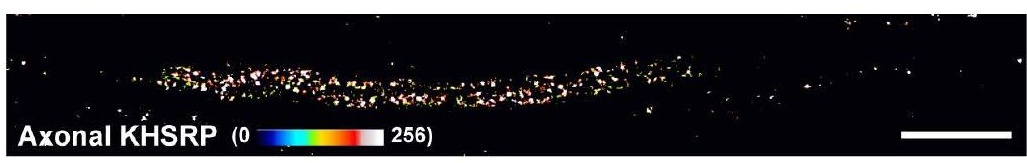


Figure 2

$\Delta$
$\frac{\Phi}{2}$
$\frac{\Phi}{Z}$

$\mathrm{Khsrp}^{+/+}$

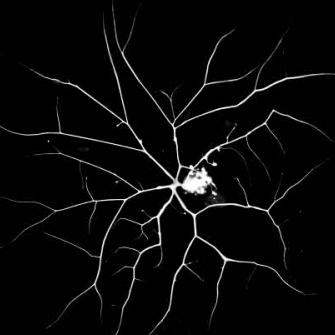

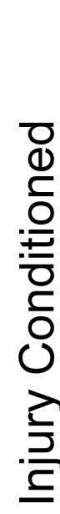

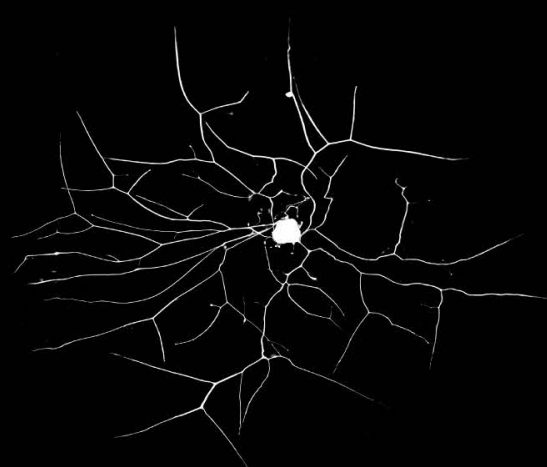

$K_{h s r p}{ }^{-1-}$
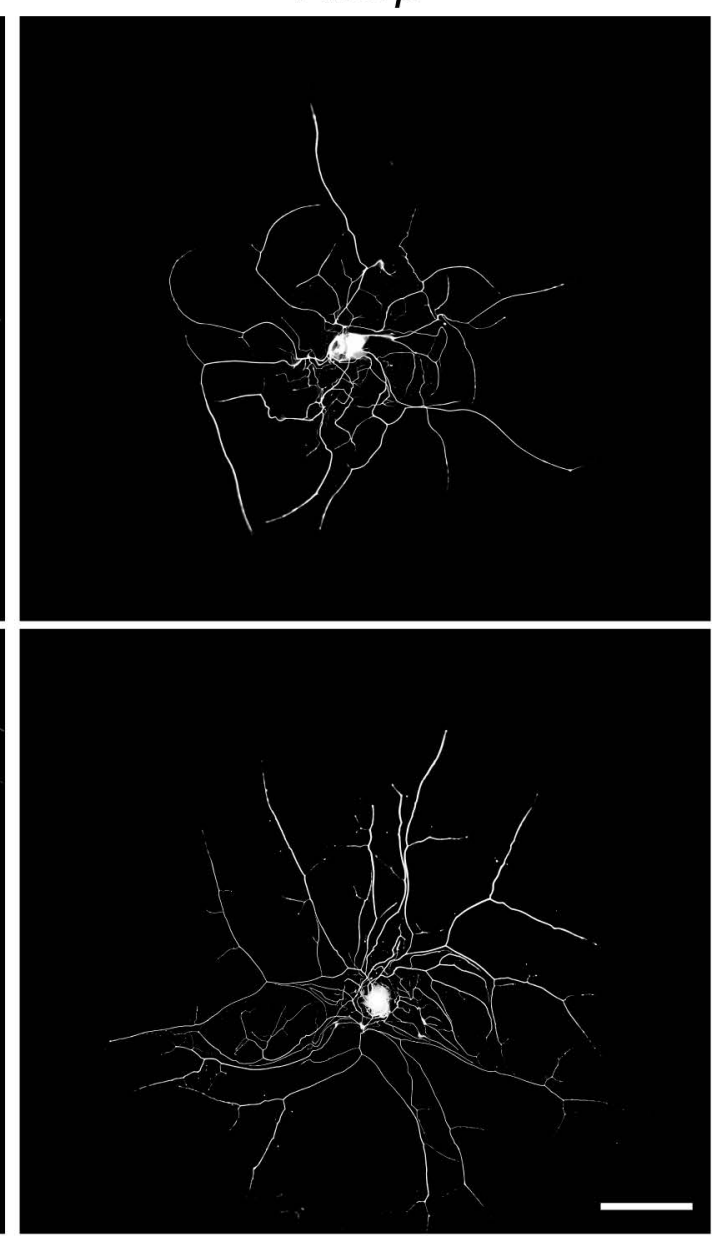

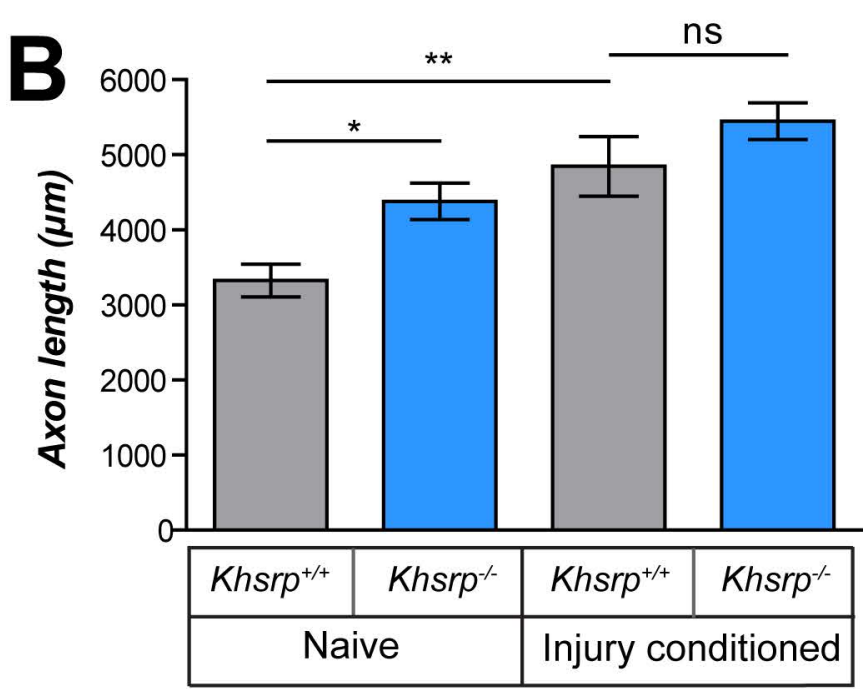

C

\begin{tabular}{|c|c|c|c|}
\hline \multicolumn{2}{|c|}{$K h s r p^{+/+}$} & \multicolumn{2}{c|}{$K h s r p^{--}$} \\
\hline $\begin{array}{c}\text { Cell } \\
\text { body }\end{array}$ & Axon & $\begin{array}{c}\text { Cell } \\
\text { body }\end{array}$ & Axon \\
\hline
\end{tabular}

KHSRP -

GAPDH - 


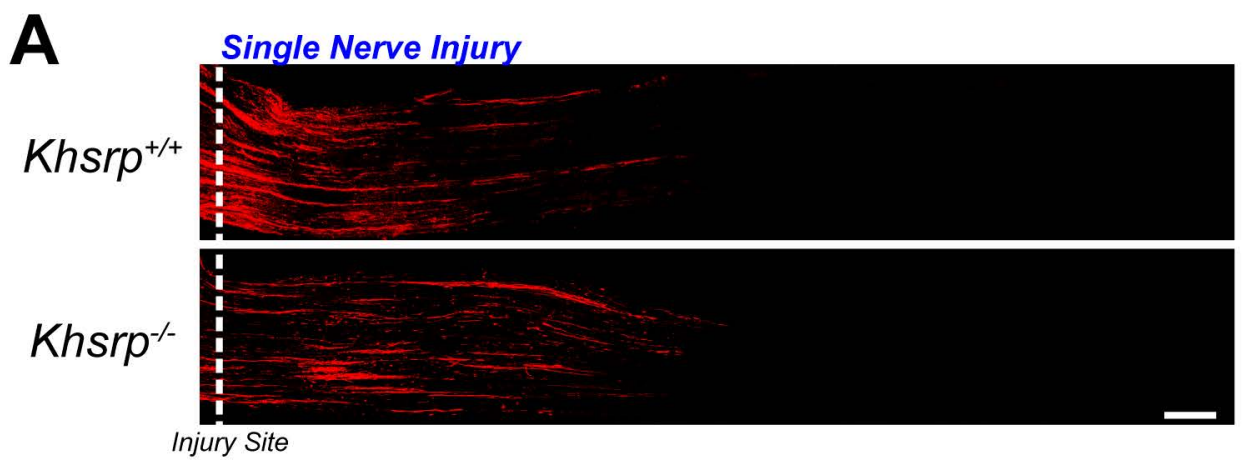

\section{Injury-conditioned Nerve}

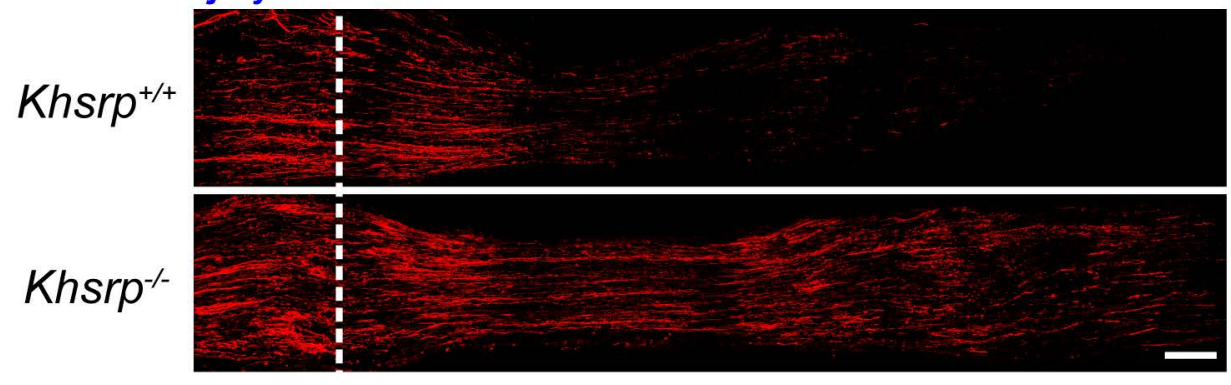

Injury Site

C Post-synaptic marker (green)
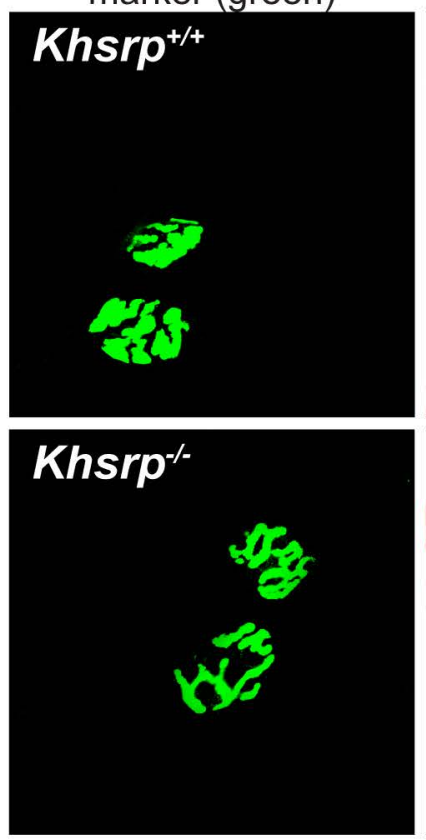

Pre-synaptic markers (red)

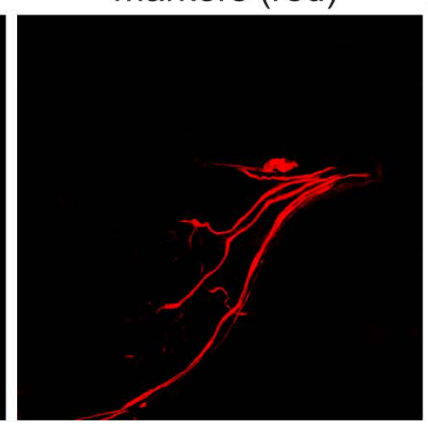

Post-syanptic (green) \& Presynaptic (red) markers
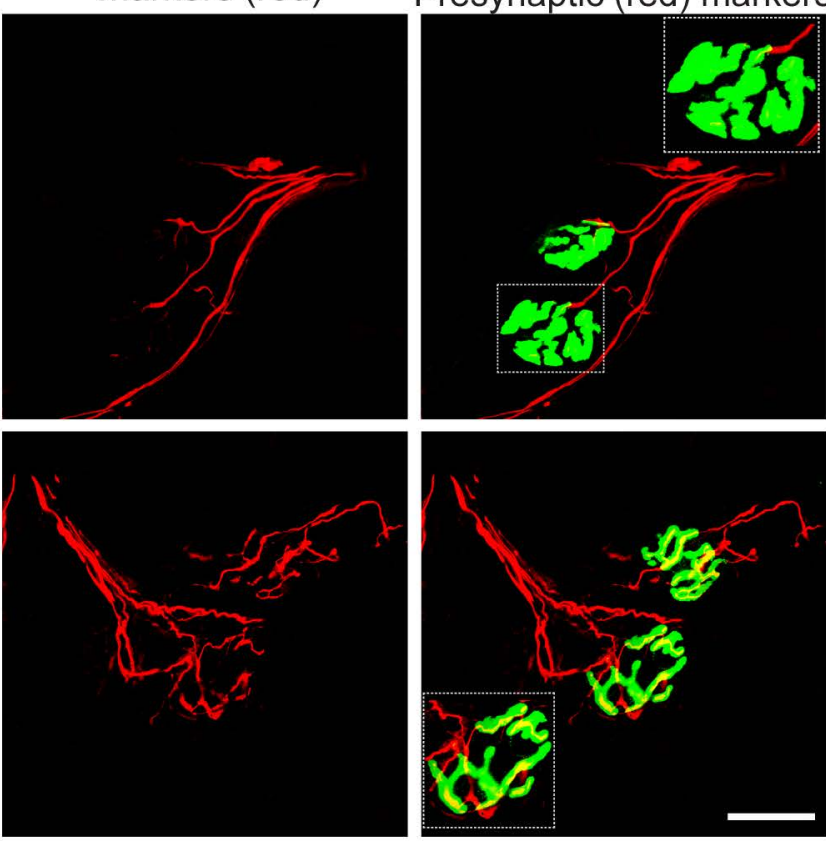

\section{E in vitro Axon}

E Regneration Assay

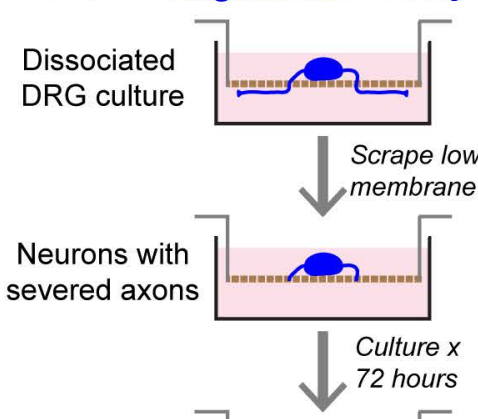

in vitro

regenerating axons
$B$ Single Nerve Injury

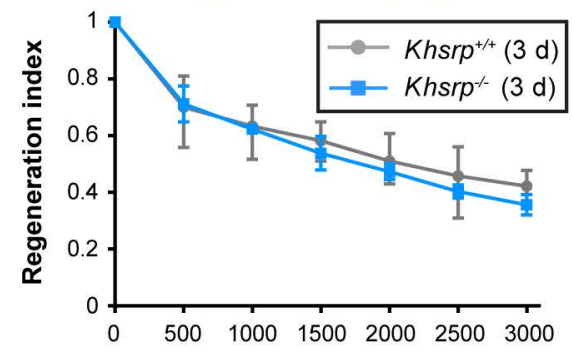

Injury-conditioned Nerve
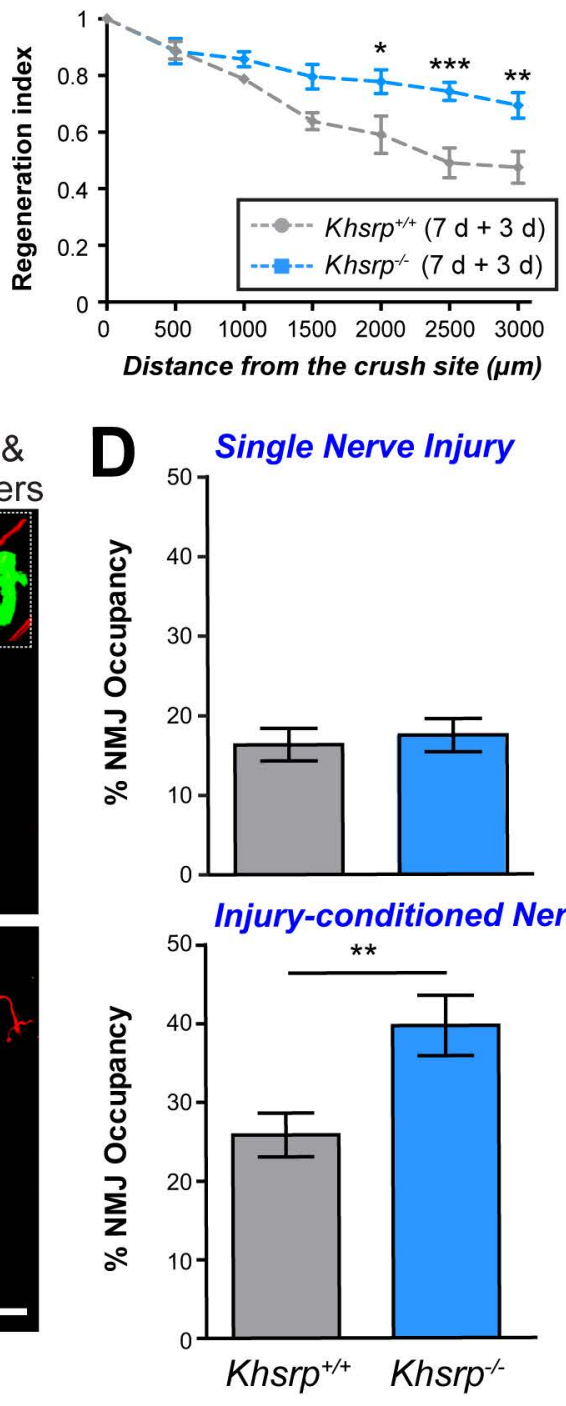

G in vitro Axon Regneration

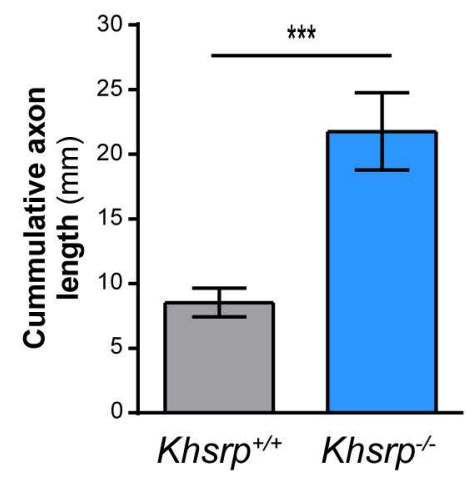


A

\section{Nerve ligation model}

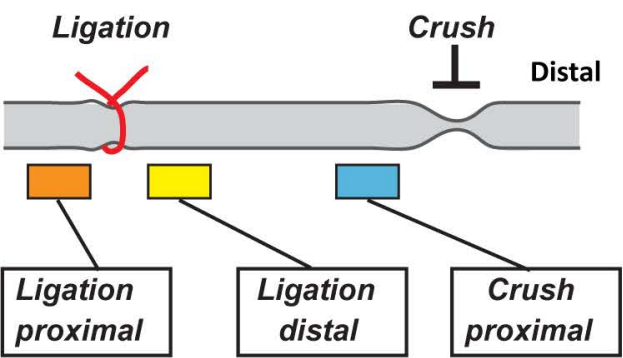

Naive

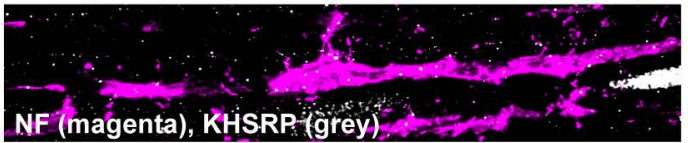

NF (magenta), KHSRP (grey)

Crush $\times 3$ hrs

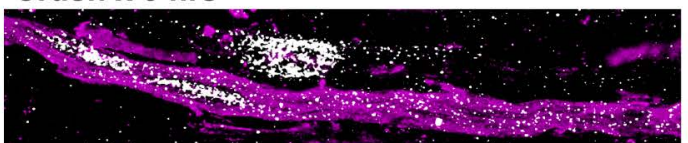

Crush $\times 16$ hrs

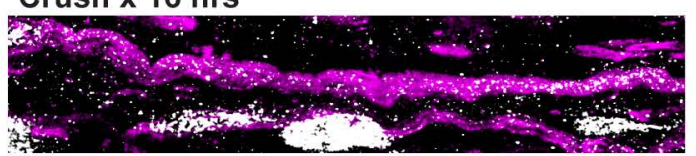

Axonal KHSRP (0 256)
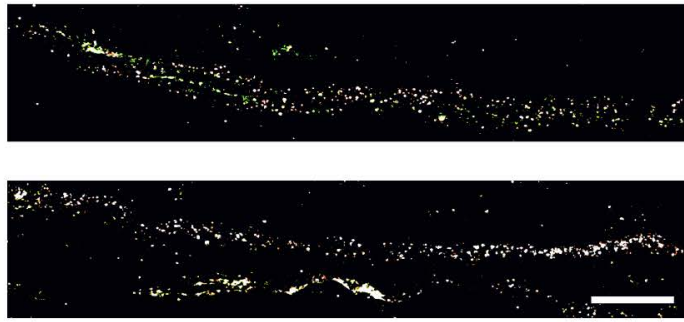

C

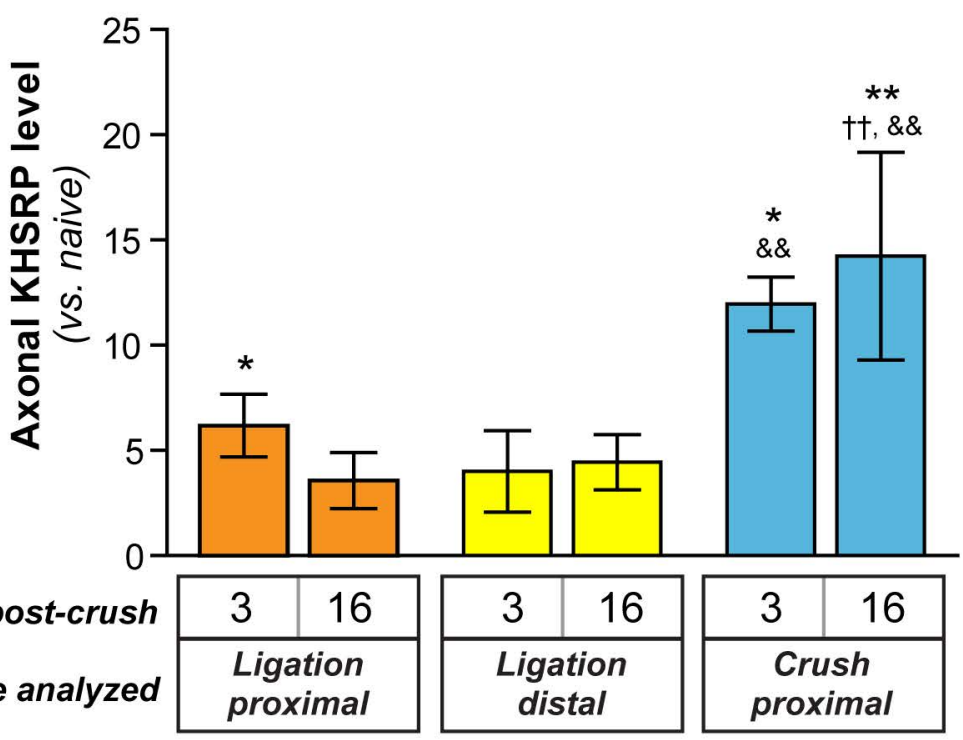

D

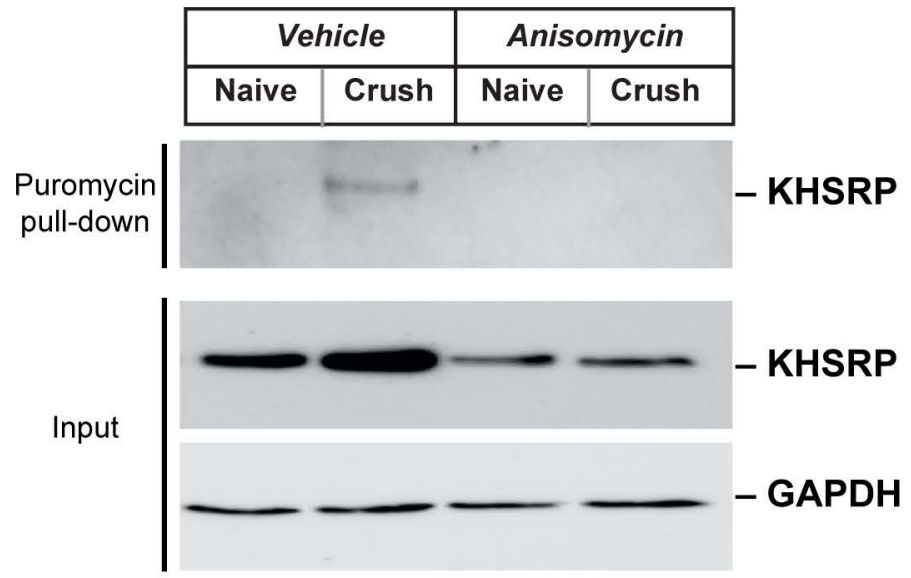

5' khsrp

3' khsrp

E

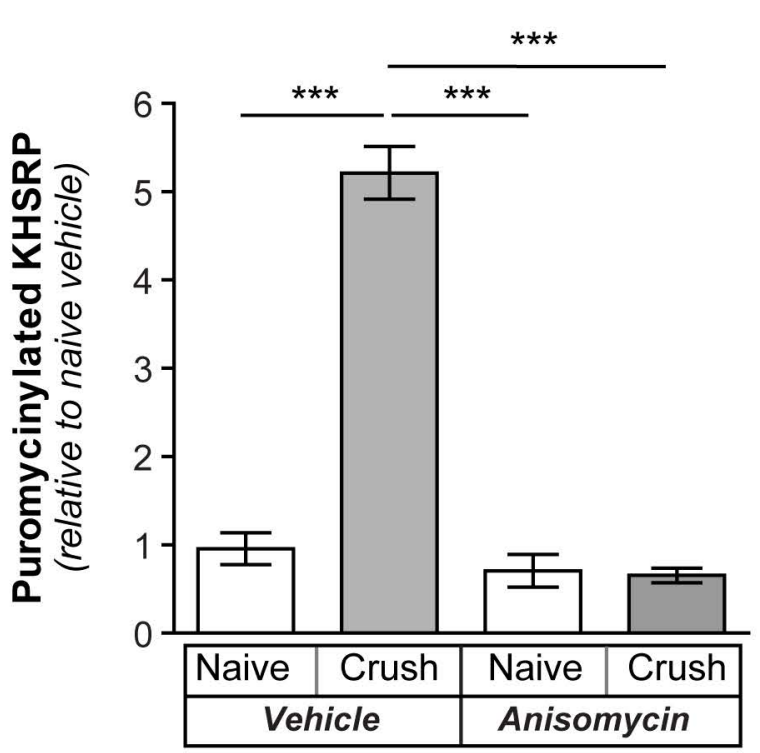

$\mathbf{F}$

GFPMYR5'/3'khsrp MMNMYR

eGFP

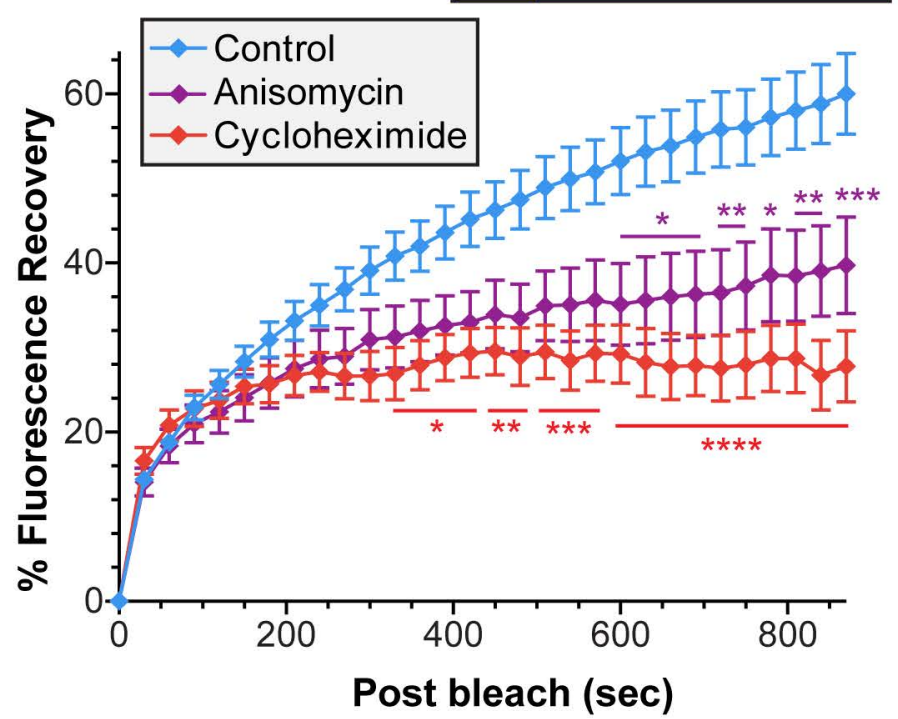


$\mathbf{A}$

Gap43 mRNA

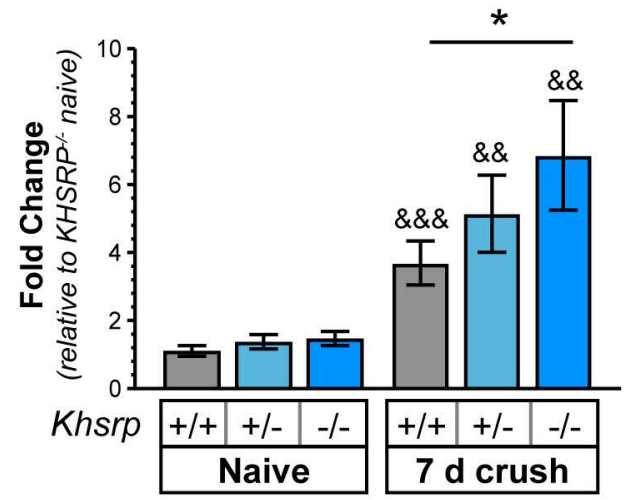

\section{GFP}

D

$K_{h s r p}^{+/+}$

Khsrp $^{-1}$

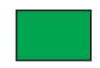

B

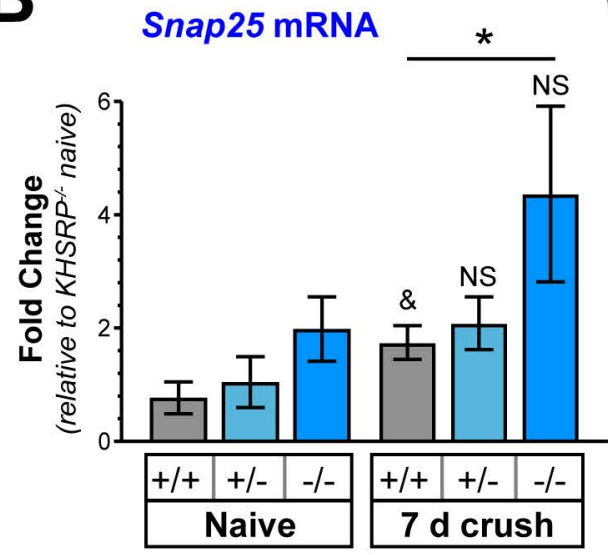

GFP-KHSRP
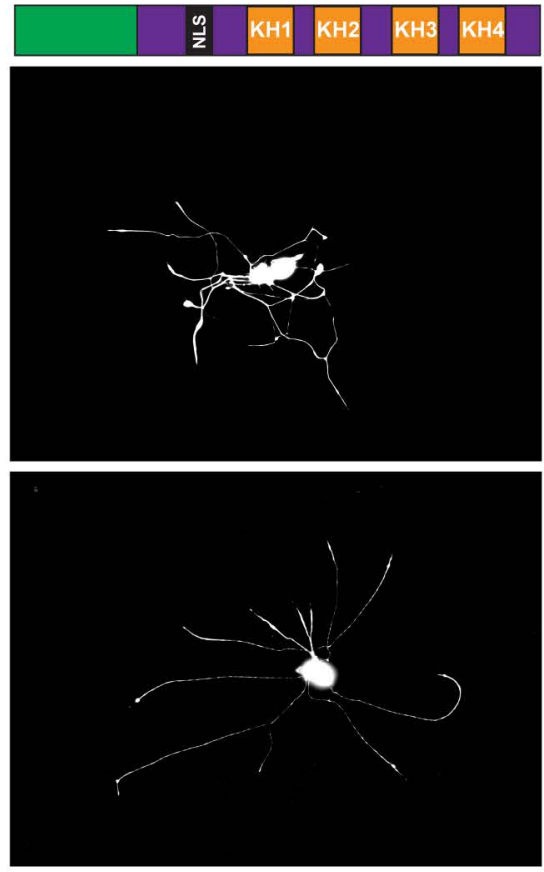

Hmgb1 mRNA

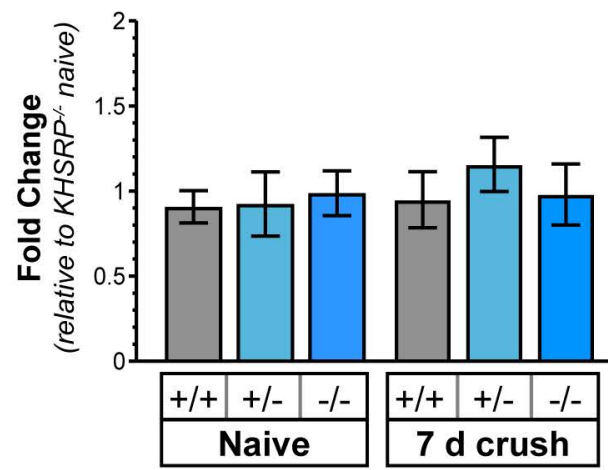

\section{GFP-KHSRP $\triangle K H 4$}

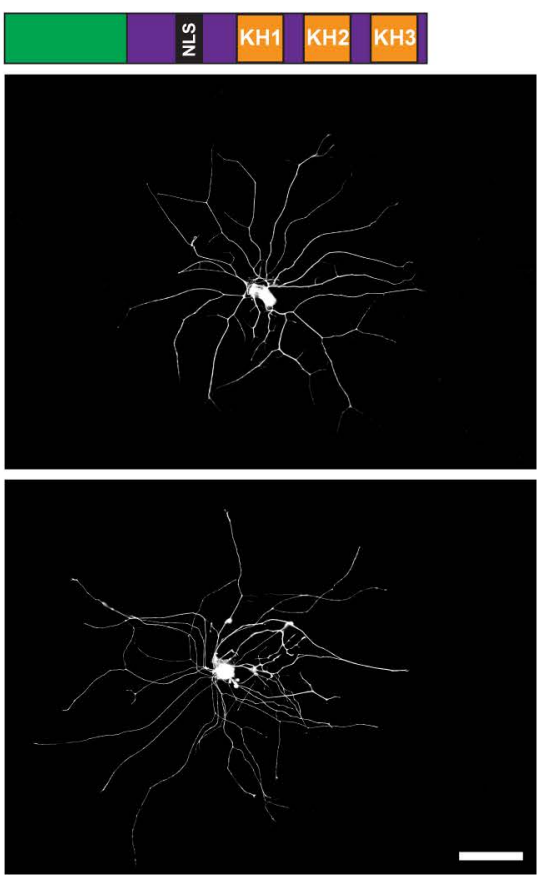

E Axon growth

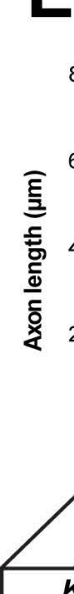

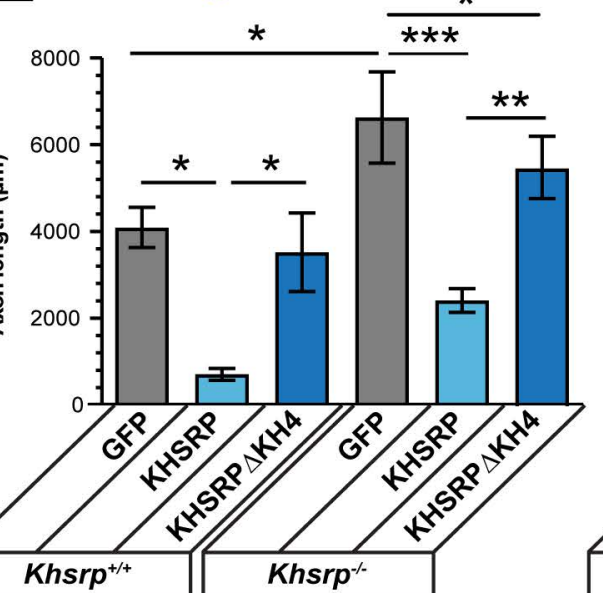

F Axonal Gap43 mRNA

G Cell body Gap43 mRNA
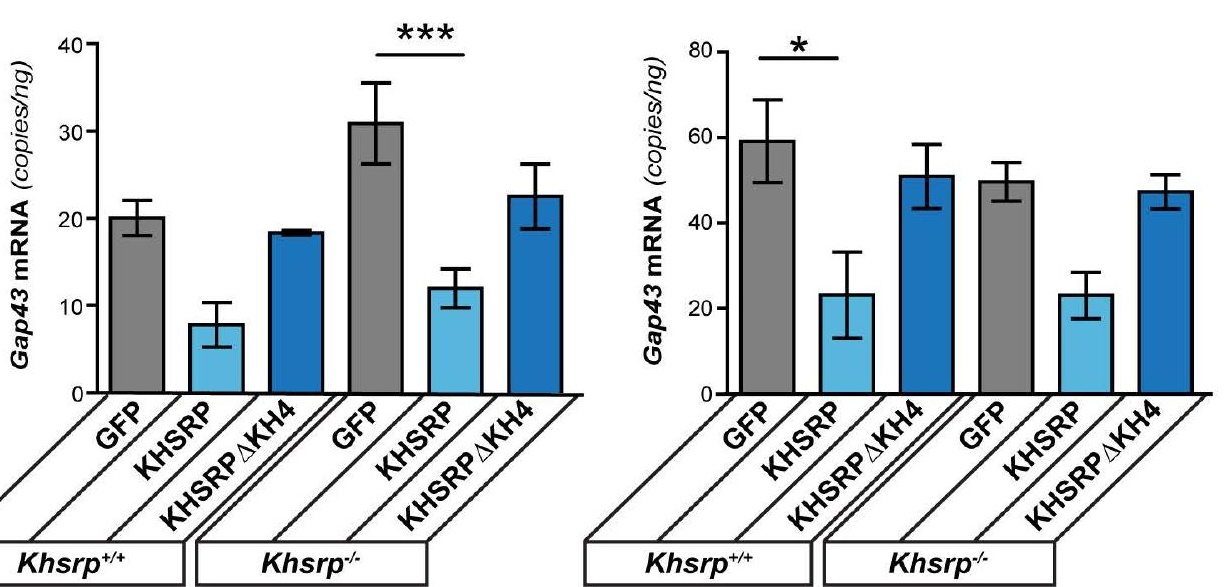